\title{
ABORDAGEM INTERTEMPORAL DA CONTA CORRENTE: INTRODUZINDO CÂMBIO E JUROS NO MODELO BÁSICO*
}

\author{
Nelson da Silva ${ }^{\S}$ \\ Joaquim Pinto de Andrade
}

\begin{abstract}
RESUMO
O modelo padrão da abordagem intertemporal da conta corrente assume as hipóteses restritivas de que todos os bens são comercializáveis e que a taxa de juros internacional é constante. Neste artigo se segue o modelo de Bergin e Sheffrin (2000) para avaliar a dinâmica das transações correntes e a mobilidade de capitais no Brasil. O modelo BS percorre a linha dos modelos de valor presente e possibilita a introdução da taxa de juros e da taxa de câmbio. Os testes do modelo seguem a técnica econométrica desenvolvida por Campbell (1987) e Campbell e Shiller (1987). Os resultados empíricos sugerem que a introdução dos juros e do câmbio no modelo intertemporal não é suficiente para que se consiga explicar consistentemente as flutuações no saldo das transações correntes do Brasil.
\end{abstract}

Palavras-chave: conta corrente, mobilidade de capitais, taxa de juros e câmbio, VAR.

\section{ABSTRACT}

The standard model of the intertemporal approach of current account assumes restrictive hypothesis that all goods are tradables and that foreign interest rate is constant. In this paper we follow Bergin and Sheffrin's (2000) model to evaluate the dynamic of the current account and the capital mobility in Brazil. BS model follows the line of the present value models and it enables us to insert interest and exchange rates. Tests of the model follow the econometric technique developed by Campbell (1987) and Campbell and Shiller (1987). The empirical results of this work suggest that the introduction of the interest and exchange rates in the intertemporal model is not enough for one to consistently explain the fluctuations of the Brazilian current account balance.

Key words: current account, capital mobility, interest and exchange rates, VAR.

Jel Classification: E27, F32, F41.

\footnotetext{
* Os autores gostariam de agradecer os comentários de um parecista anônimo e, ao mesmo tempo, isentá-lo da responsabilidade por eventuais erros remanescentes.

$\S \quad$ Universidade Federal de Viçosa - Centro de Ciências Humanas, Letras e Artes - CCH Departamento de Economia. Avenida P. H. Rolfs s/n - Campus - UFV - CEP 36570-000 - Viçosa - MG - tel: (31) 3899-1541; 3892-4496. E-mail: nelson_silva@ufv.br.

a Universidade de Brasília - Departamento de Economia. UnB - Campos Universitário Darcy Ribeiro - ICC-A $\overline{l a}$ Norte - CEP: 70910-900 - Brasília - DF - tel: (61) 3307-2498 - fax: (61) 3340-2311.E-mail: jandrade@unb.br.

Recebido em outubro de 2006. Aprovado em maio de 2007.
} 


\section{INTRODUÇão}

A conta corrente do balanço de pagamentos pode ser vista como o excedente da poupança nacional sobre o investimento. A conta corrente é fundamentalmente um fenômeno intertemporal e revela as deliberações de consumo e poupança de uma nação. $\mathrm{O}$ modelo intertemporal, por sua vez, possibilita o estudo das decisões de poupança de um agente representativo que visa estabilizar o consumo temporalmente. A teoria intertemporal considera a conta corrente como ferramenta doméstica, a qual os residentes usam para nivelar o consumo em resposta aos diversos choques econômicos.

O modelo intertemporal simples enuncia que os agentes domésticos tentam suavizar o consumo por meio de operações de empréstimos no mercado financeiro internacional. Quando se espera que a renda futura aumente em razão de um choque tecnológico específico ao país, por exemplo, os agentes domésticos procuram estabilizar o consumo tomando empréstimos no mercado financeiro internacional; por conta disso, a economia experimenta déficit em conta corrente. Por outro lado, os agentes concedem empréstimos ao exterior quando ocorre um aumento temporário na produção corrente, resultando em melhora na conta externa.

De acordo com a teoria, o saldo em transações correntes equivale ao negativo das variações esperadas no produto líquido. O procedimento padrão nessa literatura para se calcularem tais variações esperadas tem sido o uso de uma auto-regressão vetorial (VAR), envolvendo a conta corrente e o produto líquido. O modelo pode ser testado formalmente por meio de um teste Wald e informalmente por meio de uma comparação gráfica entre a conta corrente observada e os valores previstos pelo VAR.

Do ponto de vista econométrico, os resultados empíricos da abordagem intertemporal da conta corrente mostram que o modelo não se apresenta adequado para as economias pequenas, cujas variações em investimento e poupança não afetam a taxa de juros mundial. Uma explicação potencial para esse resultado, como argumentam Bergin e Sheffrin (2000), reside no fato de que as economias pequenas podem ser afetadas por choques externos, os quais não são considerados no modelo simples.

Portanto, para modelar o comportamento da conta corrente é importante incluir choques que se originam nos parceiros comerciais da nação e também distúrbios que acontecem no mundo em geral. Pode-se esperar que choques externos afetem significativamente a economia mediante movimentos na taxa de câmbio e na taxa de juros real. Pode-se afirmar que a conta corrente também serve como instrumento para um país reagir aos movimentos observados na taxa de juros mundial que são decorrentes de variações no mercado internacional de capitais.

Mudanças na taxa de juros podem afastar os consumidores de comportamentos de estabilização do consumo. Acrescenta-se que, como demonstrou Dornbusch (1983) e destacaram Bergin e Sheffrin (2000), o custo de se tomarem empréstimos do resto do mundo pode ser aumentado em decorrência de elevação antecipada no preço relativo dos bens comercializáveis internacionalmente, quando tais empréstimos são pagos em unidades desses bens, o que pode provocar substituição inter e intratemporal.

Considerados os dados da economia brasileira, neste artigo se submetem ao teste do valor presente, de acordo com Campbell (1987) e Campbell e Shiller (1987), as implicações testáveis do modelo intertemporal da conta corrente de Bergin e Sheffrin (2000), no qual não se assume que 
a taxa de juros e a taxa de câmbio real permaneçam inalteradas ao longo do tempo. São usados dados anuais e trimestrais. O trabalho está dividido em 5 seções, além desta Introdução. Na Seção 2 , desenvolve-se em detalhes o modelo utilizado. A Seção 3 é reservada à apresentação do método econométrico empregado no teste do modelo. No tópico seguinte, detalham-se os dados usados para testar o modelo. Em seguida, na Seção 5, apresentam-se e discutem-se os resultados encontrados. A seção final é reservada às conclusões e às consideraçõos finais.

\section{O MODELO}

O modelo está baseado na log-linearização da equação de Euler e da restrição orçamentária intertemporal.

\subsection{O problema do consumidor}

Seguindo a especificação de Dornbusch (1983), considere-se uma pequena economia que produz bens tradables e nontradables. Deixe-se $C_{T, t}$ ser o consumo de bens tradables no período $t ; C_{N, t} \mathrm{o}$ consumo de bens nontradables; $U\left(C_{T, t} ; C_{N, t}\right)$ a função utilidade do consumidor e $\beta \in(0,1)$ o fator de desconto subjetivo. A função utilidade vitalícia do consumidor é dada por:

$$
U_{t}=E_{t}\left\{\sum_{s=t}^{\infty} \beta^{s-t} u\left(C_{T, s} ; C_{N, s}\right)\right\}
$$

$E_{t}$ é o operador de expectativa condicional ao conjunto de informação em $t$. Define-se $B_{t+1}, Q_{t}$, $I_{t}$ e $G_{t}$ como o estoque de ativos externos no fim do período $t$, o produto, o investimento e os gastos do governo no período $t$, respectivamente. Denote-se $P_{t}$ como o preço relativo do bem doméstico nontradable em termos do bem tradable e, finalmente, $r_{t}$ como a taxa de juros mundial em termos de bens tradables. A economia pode emprestar e tomar emprestado do resto do mundo a uma taxa de juros variante no tempo, determinada nos mercados financeiros internacionais. Dessa forma, tem-se a seguinte restrição orçamentária do consumidor:

$$
C A_{t}=B_{t+1}-B_{t}=Q_{t}+r_{t} B_{t}-\left(C_{T, t}+P_{t} C_{N, t}\right)-G_{t}-I_{t}
$$

A variável $C A$ representa o saldo em transações correntes.

\subsection{1 Índice de consumo e preços}

Supõe-se que a função utilidade seja dada por:

$$
u\left(C_{T, s} ; C_{N, s}\right)=\frac{1}{1-\sigma}\left(C_{T, t}^{a} C_{N, t}^{1-a}\right)^{1-\sigma}
$$

Em (3), $\sigma^{-1}=\gamma>0$ é a elasticidade de substituição intertemporal, e $a$ representa a proporção de bens tradables no consumo da família. 
Define-se um índice de consumo total na forma Cobb-Douglas, indexado por $C^{*}$, para a função utilidade:

$$
C_{t}^{*}=C_{T, t}^{a} C_{N, t}^{1-a}
$$

Um índice de preços, $P^{*}$, é definido de tal modo que o gasto total em uma unidade do índice de consumo, definido acima, seja o mínimo possível. Dessa forma, resolve-se o seguinte problema para encontrar $P^{*}$ :

$$
\begin{array}{cc}
\underset{C_{T, t}, C_{N, t}}{\operatorname{Min}} & P_{t}^{*}=C_{T, t}+P_{t} C_{N, t} \\
\text { s.a } & C_{T, t}^{a} C_{N, t}^{1-a}=1
\end{array}
$$

Substituem-se os valores de $C_{T, t}$ e $C_{N, t}$ encontrados na solução do problema acima na respectiva função objetivo para obter $P^{*}$ :

$$
P_{t}^{*}=P_{t}^{(1-a)}\left[a^{-a}(1-a)^{-(1-a)}\right]
$$

Substitui-se $C^{*}$ na função utilidade (3) e $P^{*} C^{*}$ na restrição orçamentária (2) para obter o seguinte problema de otimização do consumidor:

$$
\begin{aligned}
& \underset{\left\{C_{s}^{*}\right\}}{\operatorname{Max}} \quad U_{t}=E_{t}\left\{\sum_{s=t}^{\infty} \beta^{s-t}\left[(1-\sigma)^{-1}\left(C_{s}^{*}\right)^{1-\sigma}\right]\right\} \\
& \text { s.a } \quad B_{t+1}=\left(1+r_{t}\right) B_{t}+Q_{t}-P_{t}^{*} C_{t}^{*}-G_{t}-I_{t}
\end{aligned}
$$

\subsubsection{A restrição orçamentária intertemporal}

Para se resolver o problema (7), é necessário obter-se a restrição intertemporal. Para tanto, a iteração para frente da restrição orçamentária até o período $t+T$ resulta em:

$$
\left[\prod_{v=t+1}^{T}\left(1+r_{v}\right)\right]^{-1} B_{t+T}=B_{t+1}+\sum_{s=t+1}^{T}\left\{\left[\prod_{v=t+1}^{s}\left(1+r_{v}\right)\right]^{-1}\left(N O_{s}-C_{s}\right)\right\}
$$

Na equação (8), NO é o produto líquido, definido como $Q-G-I$, e $C$ corresponde à $P^{*} C^{*}$. Impõe-se a condição de transversalidade:

$$
\lim _{T \rightarrow \infty}\left[\prod_{v=t+1}^{T}\left(1+r_{v}\right)\right]^{-1} B_{t+T}=0
$$

Usada a condição de transversalidade, resolve-se (8) para $B_{t+1}$, substitui-se na restrição orçamentária do problema (7) e toma-se a esperança para obter:

$$
E_{t}\left\{\sum_{s=t}^{\infty} R_{t, s} C_{s}\right\}=E_{t}\left\{\sum_{s=t}^{\infty} R_{t, s} N O_{s}\right\}+B_{t}
$$

onde, 


$$
R_{t, s} \equiv\left\{\begin{array}{lll}
{\left[\prod_{v=t+1}^{s}\left(1+r_{v}\right)\right]^{-1}} & \text { se } & s \geq t+1 \\
1 & \text { se } & s=t
\end{array}\right.
$$

\subsubsection{A equação de Euler}

De forma geral, a condição de primeira ordem para o problema do consumidor (7), considerando a restrição orçamentária intertemporal (10), é:

$$
u^{\prime}\left(C_{t}^{*}\right)=\beta^{s-t} E_{t}\left\{R_{t, s}\left(P_{t}^{*} / P_{s}^{*}\right) u^{\prime}\left(C_{s}^{*}\right)\right\} \quad \forall s>t
$$

Em particular, para $C_{t}^{*}$ e $C_{t+1}^{*}$, tem-se:

$$
E_{t}\left[\beta\left(1+r_{t+1}\right)\left(\frac{P_{t}^{*}}{P_{t+1}^{*}}\right)\left(\frac{C_{t}^{*}}{C_{t+1}^{*}}\right)^{\sigma}\right]=1
$$

Para estimação, substitui-se o índice de preços $P_{t}^{*}$ dado em (6) na equação de Euler:

$$
E_{t}\left[\beta\left(1+r_{t+1}\right)\left(\frac{C_{t}}{C_{t+1}}\right)^{\sigma}\left(\frac{P_{t}}{P_{t+1}}\right)^{(1-\sigma)(1-a)}\right]=1
$$

\subsection{Log-linearização da equação de Euler e da restrição orçamentária intertemporal}

\subsubsection{Equação de Euler}

Como mencionado por Campbell, Lo e MacKinlay (1997), uma variável X que tenha distribuição condicional log-normal e seja homocedástica apresenta as seguintes propriedades:

$$
\begin{aligned}
& \log E_{t} X=E_{t}[\log X]+\frac{1}{2} \operatorname{Var}_{t}[\log X] \\
& \operatorname{Var}_{t}[\log X]=E\left[\left(\log X-E_{t}[\log X]\right)^{2}\right]=\operatorname{Var}\left[\log X-E_{t}[\log X]\right] \\
& \text { Faz-se } X=E_{t}\left[\beta\left(1+r_{t+1}\right)\left(\frac{C_{t}}{C_{t+1}}\right)^{\sigma}\left(\frac{P_{t}}{P_{t+1}}\right)^{(1-\sigma)(1-a)}\right], \text { assumindo log-normalidade e tam- }
\end{aligned}
$$

bém que as variâncias e as co-variâncias não variem no tempo, e pode-se escrever 


$$
\begin{aligned}
\log E_{t} X=E_{t} & {\left[\log \beta+\log \left(1+r_{t+1}\right)+\sigma \log \left(\frac{C_{t}}{C_{t+1}}\right)+(1-\sigma)(1-a) \log \left(\frac{P_{t}}{P_{t+1}}\right)\right]+} \\
& +\frac{1}{2} \operatorname{Var}\left\{\log \beta+\log \left(1+r_{t+1}\right)+\sigma \log \left(\frac{C_{t}}{C_{t+1}}\right)+(1-\sigma)(1-a) \log \left(\frac{P_{t}}{P_{t+1}}\right)-\right. \\
& \left.-E_{t}\left[\log \beta+\log \left(1+r_{t+1}\right)+\sigma \log \left(\frac{C_{t}}{C_{t+1}}\right)+(1-\sigma)(1-a) \log \left(\frac{P_{t}}{P_{t+1}}\right)\right]\right\}
\end{aligned}
$$

Define-se $r_{t+1}=\log \left(1+r_{t+1}\right) ; \Delta c_{t+1}=\log C_{t+1}-\log C_{t} ; \Delta p_{t+1}=\log P_{t+1}-\log P_{t}$. Dessa forma, a expressão (14) pode ser aproximada como:

$$
\begin{aligned}
& 0=\log \beta+E_{t}\left[r_{t+1}-\sigma \Delta c_{t+1}-(1-\sigma)(1-a) \Delta p_{t+1}\right]+ \\
& +\frac{1}{2} \operatorname{Var}\left\{\left(r_{t+1}-E_{t} r_{t+1}\right)-\sigma\left(\Delta c_{t+1}-E_{t} \Delta c_{t+1}\right)-(1-\sigma)(1-a)\left(\Delta p_{t+1}-E_{t} \Delta p_{t+1}\right\}\right.
\end{aligned}
$$

Ao se notar a definição de variância acima e escrever $\omega_{X}^{2} ; \omega_{X, Y}$ como a variância de $X$ e a co-variância de $X, Y$, respectivamente, finalmente se encontra a aproximação log-linear da equação de Euler:

$$
\begin{aligned}
E_{t} \Delta c_{t+1}= & \gamma E_{t}\left[r_{t+1}+\frac{(1-\gamma)}{\gamma}(1-a) \Delta p_{t+1}\right]+\log \beta+ \\
+ & \frac{1}{2}\left\{\omega_{c}^{2}+\gamma^{2} \omega_{r}^{2}+(1-\gamma)^{2}(1-a)^{2} \omega_{p}^{2}+\right. \\
& \left.+2 \gamma \omega_{c, r}+2(1-\gamma)(1-a) \omega_{c, p}+2 \gamma(1-\gamma)(1-a) \omega_{r, p}\right\}
\end{aligned}
$$

\subsubsection{Restrição orçamentária}

A log-linearização da restrição orçamentária intertemporal (10) segue Huang e Lin(1993), que, por sua vez, se baseiam no método de log-linearização de Campbell e Mankiw (1989). Definese a restrição intertemporal (10) como:

$$
\Phi_{t}=\Psi_{t}+B_{t}
$$

onde, $\Phi_{t}=\sum_{s=t}^{\infty} R_{t, s} C_{s} ; \Psi_{t}=\sum_{s=t}^{\infty} R_{t, s} N O_{s}$.

A restrição pode ser log-linearizada em três passos. $\mathrm{O}$ primeiro consiste em log-linearizar o valor presente do produto líquido $N O$ corrente e futuro:

$$
\Psi_{t}=\sum_{s=t}^{\infty} R_{t, s} N O_{s}
$$

A equação acima (19) implica que a lei de movimento para $\Psi_{t}$ é dada por:

$$
\Psi_{s+1}=\left(1+r_{s+1}\right)\left(\Psi_{s}-N O_{s}\right) ; \quad \forall s \geq t
$$

1 De acordo com Campbell, Lo e MacKinlay (1997), Hansen e Singleton (1983) foram os primeiros a derivar uma equação de Euler similar à (17); a equação (14) foi pioneiramente apresentada por Grossman e Shiller (1981). 
Dividem-se os dois lados da equação (20) por $\Psi_{s}$ e toma-se o logaritmo natural:

$$
\psi_{t+1}-\psi_{t}=r_{t+1}+\log \left(1-\frac{N O_{t}}{\Psi_{t}}\right)=r_{t+1}+\log \left(1-e^{n O_{t}-\psi_{t}}\right)
$$

Procede-se a uma aproximação de primeira ordem de Taylor na parte não-linear da equação (21) em torno do ponto $\frac{\Psi_{t}}{N O_{T}}=\frac{\Psi}{N O}$ e substitui-se nessa mesma equação para obter: ${ }^{2}$

$$
\psi_{t+1}-\psi_{t} \approx r_{t+1}+\kappa+\left(1-\frac{1}{\rho}\right)\left(n o_{t}-\psi_{t}\right)
$$

Na equação anterior, o parâmetro $\rho=1-\exp ($ no- $\psi)$ é um número menor do que 1 e a constante $\kappa=\log (\rho)-(1-1 / \rho) \log (1-\rho)$.

Note-se que:

$$
\psi_{t+1}-\psi_{t}=\Delta n o_{t+1}+\left(n o_{t}-\psi_{t}\right)-\left(n o_{t+1}-\psi_{t+1}\right)
$$

Substitui-se (23) em (22) e rearranjam-se os termos para chegar à seguinte equação em diferenças:

$$
n o_{t}-\psi_{t}=\rho\left(r_{t+1}-\Delta n o_{t+1}\right)+\rho\left(n o_{t+1}-\psi_{t+1}\right)+\rho \kappa
$$

Resolve-se (24) iterativamente para frente e chega-se ao resultado final:

$$
n o_{t}-\psi_{t}=\sum_{s=t+1}^{\infty} \rho^{s-t}\left(r_{s}-\Delta n o_{s}\right)+\xi
$$

onde $\xi$ é uma constante.

O segundo passo consiste na log-linearização do valor presente do consumo:

$$
\Phi_{t}=\sum_{s=t}^{\infty} R_{t, s} C_{s}
$$

Note-se a semelhança com a equação do valor presente do produto líquido (19). Segue-se o mesmo procedimento realizado anteriormente e chega-se à seguinte equação:

$$
c_{t}-\phi_{t}=\sum_{s=t+1}^{\infty} \rho^{s-t}\left(r_{s}-\Delta c_{s}\right)+\xi
$$

Na equação (27) foi feita a hipótese simplificadora de que $\rho=1-\frac{C}{\Phi}$.

O terceiro e último passo corresponde à log-linearização da restrição orçamentária expressa na equação (18), que pode ser escrita como $\Psi_{t}=\Phi_{t}-B_{t}$. Divide-se por $\Phi_{t}$ e toma-se o logaritmo dos dois lados para se obter:

2 A parte não-linear da equação (21) corresponde à $\log \left(1-e^{n o_{t}-\psi_{t}}\right)$, que pode ser aproximada pelo método convencional para gerar $\kappa+\left(1-\frac{1}{\rho}\right)\left(n o_{t}-\psi_{t}\right)$. 


$$
\psi_{t}-\phi_{t}=\log \left(1-e^{\left(b_{t}-\phi_{t}\right)}\right)
$$

Log-lineariza-se a parte não-linear da equação (28) para obter:

$$
\begin{aligned}
& \psi_{t}-\phi_{t}=\left(1-\frac{1}{\Omega}\right)\left(b_{t}-\phi_{t}\right)+\kappa \\
& \therefore \Omega=\left(1-e^{b-\phi}\right) ; \quad \kappa=\log (\Omega)-\left(1-\frac{1}{\Omega}\right) \log (1-\Omega)
\end{aligned}
$$

Substitui-se (25) e (27) em (29), omite-se a constante $\kappa$ e toma-se a esperança para obter a restrição orçamentária intertemporal log-linearizada:

$$
-E_{t} \sum_{s=t+1}^{\infty} \rho^{s-t}\left[\Delta n o_{s}-\frac{1}{\Omega} \Delta c_{s}-\left(1-\frac{1}{\Omega}\right) r_{s}\right]=n o_{t}-\frac{1}{\Omega} c_{t}+\left(\frac{1}{\Omega}-1\right) b_{t}
$$

\subsection{A conta corrente}

A condição de Euler (17) e a restrição orçamentária intertemporal (30) determinam a conta corrente. A equação de Euler pode ser reescrita como:

$$
E_{t} \Delta c_{t+1}=\gamma E_{t} r_{t+1}^{*}
$$

onde:

$$
r_{t}^{*}=r_{t}+\frac{(1-\gamma)}{\gamma}(1-a) \Delta p_{t}+\text { constante }
$$

A equação (31) descreve a característica principal do comportamento maximizador do agente representativo. Essa condição identifica como o perfil ótimo de consumo é influenciado pela taxa de juros real composta, tal como definida. Essa taxa $r^{*}$ reflete a taxa de juros $r$ e a mudança no preço relativo do bem não-comercializável internacionalmente, $\Delta p$. O modelo simples da conta corrente não inclui essas variáveis, e o consumo segue um passeio aleatório, o que significa um perfil no qual a mudança esperada no consumo é nula, ou seja, $E_{t} C_{s}=C_{t}$ para $s>t$. Desta forma, os indivíduos sempre tentam suavizar o consumo por tomar e emprestar no mercado financeiro internacional, funcionando a conta corrente como meio pelo qual impactos transitórios sobre o produto líquido são amortecidos.

Ao contrário, como explicam Bergin e Sheffrin (2000), de acordo com (31), o consumidor pode ser induzido a alterar o perfil de consumo e, assim, não suavizá-lo diante das mudanças nos termos de empréstimos e pagamentos. Em primeiro lugar, se o agente espera aumento na taxa de juros convencional $r$, o consumo corrente torna-se mais caro em termos do consumo futuro do qual se está abrindo mão, levando-o à substituição em direção ao consumo futuro com elasticidade $\gamma$. Outro efeito intertemporal pode tornar-se possível como resultado de mudança no preço relativo dos bens não-comercializáveis no mercado internacional. Se o preço do bem tradable está temporariamente baixo e espera-se que ele aumente no futuro, o pagamento de empréstimos em bens 
tradables tem custo maior em termos da cesta de consumo que da cesta de bens comercializáveis apenas. Então, a taxa de juros $r^{*}$ é superior à taxa convencional $r$ e reduz o gasto total em consumo presente pela elasticidade $\gamma(1-a)$.

As mudanças no preço relativo dos bens nontradables também provocam substituição intratemporal. Se o preço dos bens tradables está temporariamente baixo em relação ao dos bens nontradables, as famílias substituem os bens nontradables pela elasticidade intratemporal, que é unitária em virtude da especificação Cobb-Douglas. Isso aumenta o gasto com consumo corrente pela elasticidade $(1-a)$. Lembra-se que o efeito intertemporal reduz o gasto em $\gamma(1-a)$, e o efeito intratemporal pode ser dominado pelo intertemporal se a elasticidade de substituição intertemporal $\gamma$ for maior que 1 . $^{3}$

Para encontrar uma equação para a conta corrente, insere-se a equação de Euler (31) na restrição orçamentária (30). Isso leva à seguinte equação:

$$
-E_{t} \sum_{s=t+1}^{\infty} \rho^{s-t}\left[\Delta n o_{s}-\frac{\gamma}{\Omega} r_{s}^{*}-\left(1-\frac{1}{\Omega}\right) r_{s}\right]=n o_{t}-\frac{1}{\Omega} c_{t}+\left(\frac{1}{\Omega}-1\right) b_{t}
$$

Seguido o procedimento convencional de escolher que os ativos estrangeiros líquidos são iguais a zero no estado estacionário, o que implica $\Omega$ igual a 1, a condição (33) pode ser escrita como:

$$
C A_{t}^{*}=-E_{t} \sum_{s=t+1}^{\infty} \beta^{s-t}\left[\Delta n o_{s}-\gamma r_{s}^{*}\right]
$$

Por um lado, a condição (34) tem a mesma interpretação do modelo intertemporal da conta corrente no qual a taxa de juros é considerada constante. Ou seja, em decorrência da suavização do consumo, o saldo em conta corrente diminui sempre que os agentes esperam que o produto líquido aumente. Mas a condição (34) também indica que ocorre redução na conta corrente sempre que a taxa $r^{*}$ diminui. Isso ocorre porque uma queda na taxa $r^{*}$ induz os agentes a aumentarem seu consumo em um nível acima do patamar de suavização.

\section{MÉTODo ECONOMÉTRICO}

A metodologia de Bergin e Sheffrin (2000), usada para testar a equação (34), baseia-se nos procedimentos de Campbell (1987), Sheffrin e Woo (1990) e Ghosh (1995). A idéia consiste em ampliar esses procedimentos para se considerar a variável adicional $r^{*}$. A fim de testar a restrição de que a conta corrente depende dos valores futuros esperados do produto líquido e da taxa $r^{*}$, deve-se ter uma estimativa para esse conjunto de valores esperados. Sob a hipótese nula, a conta corrente (34) deveria por si incorporar toda a informação dos agentes sobre os valores futuros da combinação linear de taxa de juros e mudanças no produto líquido especificados em (34). Ao se seguir o procedimento padrão na literatura, pode-se estimar um VAR com valores defasados da variação do produto líquido, da conta corrente e da taxa $r^{*}$. Então, pode-se construir o seguinte VAR para representar a previsão dos indivíduos:

3 No Apêndice G apresentam-se as derivações das elasticidades. 


$$
\left[\begin{array}{l}
\Delta n o_{t} \\
C A_{t}^{*} \\
r_{t}^{*}
\end{array}\right]=\left[\begin{array}{lll}
A_{11}(L) & A_{12}(L) & A_{13}(L) \\
A_{21}(L) & A_{22}(L) & A_{23}(L) \\
A_{31}(L) & A_{32}(L) & A_{33}(L)
\end{array}\right]\left[\begin{array}{l}
\Delta n o_{t-1} \\
C A_{t-1}^{*} \\
r_{t-1}^{*}
\end{array}\right]+\left[\begin{array}{l}
e_{1 t} \\
e_{2 t} \\
e_{3 t}
\end{array}\right]
$$

onde,

$A_{i j}(L)=$ polinômios no operador $L$;

$e_{i t}^{i j}=$ erros de previsão, que podem ser correlacionados.

Considera-se um VAR de ordem p; assim, a equação (35) pode ser escrita de forma mais compacta, de tal forma que:

$$
\mathbf{z}_{t}=\mathbf{A}_{1} \mathbf{z}_{t-1}+\mathbf{A}_{2} \mathbf{z}_{t-2}+\cdots+\mathbf{A}_{p} \mathbf{z}_{t-p}+\mathbf{e}_{t}
$$

Na equação anterior, tem-se $\mathbf{z}_{t}=\left(\Delta n o_{t} ; C A_{t}^{*} ; r_{t}^{*}\right)$.

O VAR acima pode ser transformado num VAR(1) de tal modo que:

$$
\mathbf{x}_{t}=\mathbf{A} \mathbf{x}_{t-1}+\mathbf{v}_{t} \quad \Rightarrow E\left(\mathbf{x}_{s}\right)=\mathbf{A}^{s-t} \mathbf{x}_{t}
$$

onde,

$$
\mathbf{x}_{t}=\left[\begin{array}{c}
\mathbf{z}_{t} \\
\mathbf{z}_{t-1} \\
\vdots \\
\mathbf{z}_{t-p+1}
\end{array}\right] ; \quad \mathbf{A}=\left[\begin{array}{cccccc}
\mathbf{A}_{1} & \mathbf{A}_{2} & \mathbf{A}_{3} & \cdots & \mathbf{A}_{p-1} & \mathbf{A}_{p} \\
\mathbf{I}_{3} & \mathbf{0} & \mathbf{0} & \cdots & \mathbf{0} & \mathbf{0} \\
\mathbf{0} & \mathbf{I}_{3} & \mathbf{0} & \cdots & \mathbf{0} & \mathbf{0} \\
\vdots & \vdots & \vdots & \cdots & \vdots & \vdots \\
\mathbf{0} & \mathbf{0} & \mathbf{0} & \cdots & \mathbf{I}_{3} & \mathbf{0}
\end{array}\right] ; \quad \mathbf{v}_{t}=\left[\begin{array}{c}
\mathbf{e}_{t} \\
\mathbf{0} \\
\vdots \\
\mathbf{0}
\end{array}\right] .
$$

Ao se pré-multiplicar (37) pelo vetor $\mathbf{h}=\left[\begin{array}{lllllll}1 & 0 & -\gamma & 0 & 0 & \cdots & 0\end{array}\right]$, onde $\mathbf{h}$ é de ordem 1x3p, e substituir na equação da conta corrente (34) obtém-se:

$$
C \hat{A}_{t}^{*}=-\mathbf{h} \sum_{s=t+1}^{\infty} \beta^{s-t} \mathbf{A}^{s-t} \mathbf{x}_{t}
$$

Caso se assuma que o VAR satisfaça a condição de estabilidade, ou seja, $\mathbf{A}(L)$ converge para zero quando $(s-t)$ tende a infinito, e se leve em conta que $0<\beta<1$, pode-se escrever (38) como:

$$
C \hat{A}_{t}^{*}=\mathbf{k x}_{t}
$$

onde,

$$
\mathbf{k}=-\mathbf{h} \beta \mathbf{A}[\mathbf{I}-\beta \mathbf{A}]^{-1}
$$


A expressão acima fornece um modelo de previsão da variável conta corrente consistente com o VAR e as restrições do modelo intertemporal. Note-se que $\mathbf{k x}_{t}$ não é uma previsão da conta corrente no sentindo convencional, mas apenas representação das restrições do modelo.

Se as restrições da teoria forem consistentes com os dados de tal sorte que $C \hat{A}_{t}^{*}=C A_{t}^{*}$, então o vetor $\mathbf{k}$ deve ser igual a $\mathbf{k}=\left[\begin{array}{lllll}0 & 1 & 0 & \cdots & 0\end{array}\right]$. Nesse caso, (39) pode ser escrita como:

$$
\mathbf{g} \mathbf{x}_{\mathbf{t}}=-\mathbf{h} \beta[\mathbf{I}-\beta \mathbf{A}]^{-1} \mathbf{x}_{\mathbf{t}}
$$

onde $\mathrm{g}$ é um vetor linha com $3 p$ elementos nulos, exceto o segundo, ou seja, $\mathrm{g}=\left[\begin{array}{lllll}0 & 1 & 0 & \ldots\end{array}\right]$. A equação (41) implica que se deve ter as seguintes condições:

$$
\left\{\begin{array}{l}
a_{2 j}=a_{1 j}-\gamma a_{3 j} ; \quad j=(1,3,4, \ldots, 3 p) ; \quad(j \neq 2) \\
a_{22}-a_{12}+\gamma a_{32}=1 / \bar{\beta}
\end{array}\right.
$$

Essas restrições podem ser testadas, usando-se um teste Wald.

\section{DADOS E VALORES DOS PARÂMETROS}

Para se testar a equação da conta corrente (34), empregaram-se dados trimestrais (1991:12004:1) e anuais (1947-2003) para o Brasil. Os dados foram obtidos da base de dados do Ipeadata, IBGE e da versão on-line do International Financial Statistcs (IFS).

A taxa de juros mundial $r$ foi obtida seguindo-se o procedimento de Barro e Sala-i-Marin (1990) e Bergin e Sheffrin (2000). As taxas de juros nominais de curto prazo foram obtidas com o IFS para o grupo dos sete países mais industrializados do mundo - G-7. A taxa de inflação para cada um desses países foi calculada utilizando-se o respectivo IPC. Para obtenção da inflação esperada, estimou-se um processo auto-regressivo de quarta ordem, AR(4), da inflação calculada anteriormente. Feito isso, subtraíram-se as taxas de inflação esperadas das taxas de juros nominais dos países do G-7 e obtiveram-se as respectivas taxas de juros reais ex-ante. Finalmente, chegouse à taxa de juros real mundial, calculando a média ponderada dessas taxas de juros reais; para a ponderação foi considerada a participação do PIB de cada país no total dos países do G-7 em cada período respectivo.

Para construir a série do produto líquido $(N O=Q-I-G)$, recorreu-se aos dados do Ipeadata. As séries nominais foram convertidas para valores reais, usando-se o respectivo deflator no caso dos dados trimestrais; para o período anual, todas as séries foram deflacionadas pelo deflator do PIB, pois não existe um deflator particular para cada série. A série do produto líquido foi convertida a valor per capita, sendo que, no tocante à série trimestral, obteve-se a população total por meio de interpolação. Ao tomar o logaritmo natural do produto líquido e a primeira diferença do logaritmo, alcançou-se $\Delta n o_{t}$. A série da conta corrente, $C A^{*}$, foi obtida ao se subtrair o logaritmo do consumo real per capita de $\Delta n o_{t}$. Portanto, a série construída da conta corrente é apenas uma aproximação.

No período trimestral, empregou-se, como proxy para $P_{t}$ uma medida da taxa de câmbio real fornecida pelo Ipeadata. Em relação ao horizonte 1947-2003, foi adotado o procedimento de Rogoff (1992) e Bergin e Sheffrin (2000), que utilizam taxas nominais de câmbio e as convertem 
para valores reais usando o índice de preços doméstico e o de preços ao consumidor para os países industrializados fornecidos pelo IFS do FMI. Uma apreciação esperada da taxa de câmbio real $e x-$ ante, $E_{t} \Delta p_{t+1}$, é calculada estimando-se uma auto-regressão da taxa de câmbio real de 4 períodos, com valores em logaritmo e diferenciados. Por fim, calculou-se a taxa de juros real $r{ }^{*}$ para o Brasil, ao se fazer uso taxa de juros real $r$ e da taxa de câmbio real $E_{t} \Delta p_{t+1}$, derivadas acima.

Como pode ser observado, os testes da condição (34) dependem dos valores dos parâmetros ${ }^{4} \beta$, $\alpha$ e $\gamma$. Acompanhou-se a estratégia de considerar uma região de valores para os parâmetros desconhecidos. O valor do fator de desconto $\beta$ é obtido da igualdade $\beta=1 /(1+\bar{r})$, onde $\bar{r}$ é a média amostral da taxa de juros mundial calculada anteriormente no conjunto de dados. Ao proceder dessa forma, constatou-se que o valor de $\beta$ corresponde à 0,96 para o período anual e 0,97 para o período trimestral considerado.

Em relação à participação dos bens tradables no consumo final das famílias, o conjunto de dados utilizados neste trabalho não é suficiente para sua determinação. Então, recorreu-se aos mesmos valores de Bergin e Sheffrin (2000), ou seja, $a=0,5$ e $a=2 / 3$. Essas são provenientes dos trabalhos empíricos de Stockman e Tesar (1995) e Kravis, Heston e Summers (1982).

Como assinalam Bergin e Sheffrin (2000), a elasticidade intertemporal, $\gamma$, é a mais problemática dos três parâmetros com relação à divergência de valores nos trabalhos empíricos. De acordo com Hall (1988), a elasticidade de substituição intertemporal deve se situar abaixo de 0,1, já que o consumo tende a responder fracamente à taxa de juros real. Já Mehra e Prescott (1985) sugerem $\sigma<2$, o que indica que a elasticidade de substituição intertemporal deve ser maior que 0,5. Para o caso brasileiro, Reis et al. (1998) reportam 3,6 $<\sigma<6,43(0,16<\gamma<0,28)$, enquanto Issler e Piqueira (2000) estimam a mediana de $\sigma$ como 1,7, o que acarreta elasticidade de substituição de 0,59. Em razão disso, considerou-se nos testes uma região de valores para $\gamma$, concentrando mais atenção em valores baixos para a elasticidade de substituição intertemporal, na medida em que o interesse se encontra na resposta do consumo em relação às mudanças na taxa de juros real e na taxa de câmbio real.

Antes de testar a equação da conta corrente (34), verificou-se se as variáveis envolvidas nessa expressão são estacionárias. Para examinar se as variáveis no VAR (35), $\Delta n o, C A^{*}$ e $r^{*}$, são estacionárias, foi executado o tradicional teste Dickey-Fuller aumentado (ADF) e o teste Phillips-Perron (PP). Os resultados obtidos para os dois períodos considerados são apresentados na Tabela 1.

Os resultados aludidos na Tabela 1 mostram que, para o período anual, tanto o teste ADF quanto o PP rejeitam a presença de raiz unitária nas variáveis consideradas no nível de significância de $1 \%$. Em relação aos dados trimestrais, a afirmativa anterior é válida somente para a série do produto líquido, $\Delta$ no. Apenas no nível de $10 \%$, pode-se rejeitar a presença de raiz unitária para a variável taxa de juros real composta, $r^{*}$. Quanto à conta corrente, $C A^{*}$, não se pode rejeitar a hipótese nula de raiz unitária. Dá-se prosseguimento aos demais testes para esse período, contudo, note-se que uma das condições do modelo já não foi atendida.

4 Note-se que $\beta$ e $\gamma$ também são cruciais para o teste Wald das restrições em (42). 
Tabela 1 - Teste de raiz unitária

\begin{tabular}{|c|c|c|c|c|c|c|c|}
\hline \multirow{2}{*}{ Teste } & & \multicolumn{2}{|c|}{$\begin{array}{l}\text { Mudança no Produto líquido } \\
\qquad(\Delta \mathrm{no})\end{array}$} & \multicolumn{2}{|c|}{ Conta Corrente $\left(C A^{*}\right)$} & \multicolumn{2}{|c|}{ Taxa de juros $\left(r^{*}\right)$} \\
\hline & & Anual & Trimestral & Anual & Trimestral. & Anual & Trimestra \\
\hline \multirow{2}{*}{ ADF } & Estatística $t$ & $-4.78^{\star \star \star}$ & $-11.68^{\star \star \star}$ & $-3.12^{\star \star \star}$ & -1.25 & $-3.95^{\star \star \star}$ & $-1.87^{*}$ \\
\hline & Defasagem & 0 & 2 & 0 & 0 & 10 & 4 \\
\hline \multirow{2}{*}{ PP } & Estatística $t$ & $-4.80^{\star \star \star}$ & $-13.44^{\star * *}$ & $-3.20^{* \star *}$ & -1.08 & $-3.83^{* * *}$ & $-7.91^{* *}$ \\
\hline & Defasagem & 3 & 11 & 2 & 7 & 3 & 5 \\
\hline
\end{tabular}

Notas: (i) ‘***, ‘**’ e ‘*’, indicam que a estatística t é significante no nível de $1 \%, 5 \%$ e $10 \%$, respectivamente; (ii) a amostra do período anual corresponde a 1947-2003 enquanto a do período trimestral compreende 1991-Q1 a 2004-Q1; (iii) para realizar o teste para a taxa de juros $\mathrm{r}^{*}$, usou-se a $=0,5$ e $\gamma=0,085$; (iv) assim como Bergin e Sheffrin (2000), não se incluiu tendência nem constante nos testes.

As Figuras 1 e 2 mostram o comportamento das séries da taxa de câmbio real, da taxa de juros e de $r^{*}$. O comportamento das demais séries pode ser vista em Senna e Issler (2000) e Silva e Andrade (2006). Note que, para os parâmetros utilizados, a variação da taxa de câmbio real domina o comportamento de $r^{*}$. Conforme alteram-se os parâmetros, o peso da taxa de juros aumenta/diminui. Embora não se tenha reportado o comportamento de $r$, ainda assim observa-se que essa série permanece estacionária.

Figura 1 - Variação da taxa de câmbio $\left(E_{t} \Delta p_{t+1}\right)$, taxa de juros $\left(E_{t} r_{t+1}\right)$ e $r_{t}^{*}(1947-2003)$
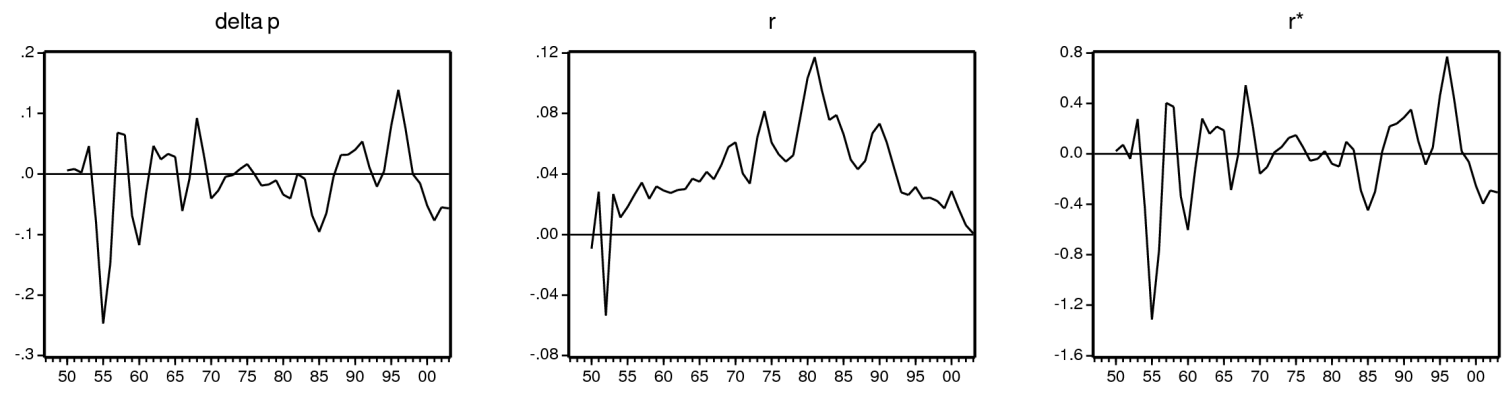


\section{Figura 2 - Variação da taxa de câmbio $\left(E_{t} \Delta p_{t+1}\right)$, taxa de juros $\left(E_{t} r_{t+1}\right)$ e $r_{t}^{*}(1991: 1-2004: 1)$}

delta $\mathrm{p}$

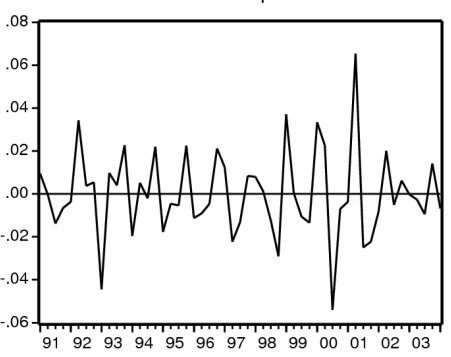

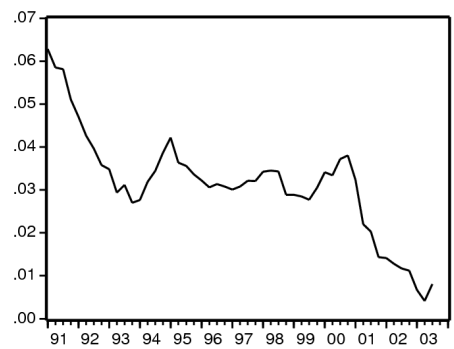

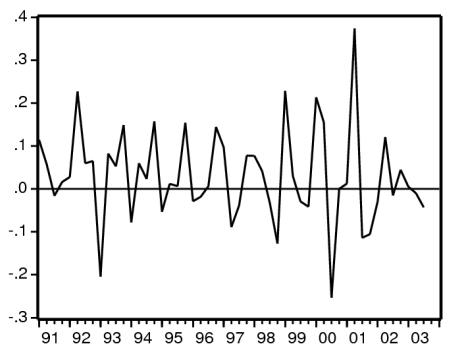

\section{RESUltados}

Os principais resultados para o período trimestral e anual são apresentados nas Tabelas 2 e 3. As Tabelas 2 e 3 possuem o mesmo formato. A primeira coluna mostra as variáveis envolvidas e a segunda os valores obtidos quando estimado o modelo básico, ou seja, sem $r^{*}$. A terceira e as demais colunas mostram o modelo incorporando a taxa de câmbio e a taxa de juros real, $r{ }^{*}$. Em cada coluna foram expostos os valores dos elementos para o vetor $\mathbf{k}$, a estatística $\chi^{2}$ e o respectivo valor-p correspondente ao teste Wald das restrições da equação (42). ${ }^{5}$ Para comparação ilustrativa, foram construídos gráficos para o modelo básico e o ampliado. Essas figuras mostram as séries reais e ajustadas da conta corrente e, portanto, o ajuste do modelo.

\subsection{Período 1991:1-2004:1}

O primeiro passo para estimar o VAR é obter a ordem apropriada. Para o modelo básico, pelo critério de Akaike - AIC, o número adequado de lags no VAR é 2. Em relação ao modelo ampliado, a ordem do VAR varia entre 2 e 3, dependendo da combinação de elasticidade de substituição e participação dos bens comercializáveis no consumo final. $\mathrm{Na}$ análise cuidadosa das propriedades dos resíduos, os testes de diagnóstico revelam que a escolha da ordem do VAR é adequada, levando-se em conta que não se encontrou autocorrelação serial, heterocedasticidade e a hipótese de normalidade dos erros não pode ser rejeitada. ${ }^{6}$

Na Tabela 2, apresentaram-se os resultados derivados tanto do modelo básico como do ampliado. No caso do modelo ampliado, no sentido de se analisar a sensibilidade, apresentaram-se dois valores diferentes para a participação dos bens tradables no consumo total privado, $a$, bem como dois valores para a elasticidade de substituição intertemporal, $\gamma$.

Os testes estatísticos apresentados na Tabela 2 rejeitam fortemente o modelo. Para o modelo básico, em que a taxa de juros mundial é constante e não se levam em conta as mudanças na taxa de câmbio real, ou seja, $r^{*}=0$, a teoria sugere que, com dois lags e duas variáveis, o vetor $\mathbf{k}$ deveria

5 O valor-p indica a probabilidade de se obter uma estatística de teste, cujo valor absoluto é maior ou igual ao valor da estatística amostral se a hipótese nula é verdadeira. Logo, valores baixos indicam a rejeição da hipótese nula. Por exemplo, se valor-p se situa entre 0.05 e 0.01 , a hipótese nula é rejeitada a 5\%, mas não no nível de $1 \%$.

6 Por restrição de espaço, não são reportados os valores encontrados para a seleção da ordem do VAR e nem as propriedades dos resíduos. 
ser ( $\left.\begin{array}{llll}0 & 1 & 0 & 0\end{array}\right)$. O coeficiente do vetor k sobre a conta corrente no período $t, C A^{*}{ }_{t}$, é - 0,225 . Esse valor não é significativamente diferente de zero e está muito distante do valor de 1 . Adicionalmente, os valores sobre o produto líquido, $\Delta n o_{t-i}, i=0,1$, e sobre os valores defasados da conta corrente são significativamente diferentes de seus valores teóricos. De modo geral, o teste Wald das restrições rejeita fortemente o modelo, dado que a estatística $\chi^{2}$ com quatro graus de liberdade é 16,567 com um valor-p de $0.002 .^{7}$

Tabela 2 - Teste do modelo intertemporal da conta corrente - 1991:1-2004:1

\begin{tabular}{|c|c|c|c|c|c|}
\hline \multirow{3}{*}{$k^{\prime}$} & \multirow{3}{*}{ Modelo básico } & \multicolumn{4}{|c|}{ Modelo ampliado } \\
\hline & & \multicolumn{2}{|c|}{$a=1 / 2$} & \multicolumn{2}{|c|}{$a=2 / 3$} \\
\hline & & $\gamma=0,085$ & $\gamma=0,5$ & $\gamma=0,085$ & $\gamma=0,5$ \\
\hline$\Delta n o_{t}$ & 0.460 & 0.386 & 0.510 & 0.388 & 0.559 \\
\hline$C A_{t}^{*}$ & -0.225 & -0.068 & -0.257 & -0.084 & -0.294 \\
\hline$r_{t}^{*}$ & - & -0.057 & 0.711 & -0.058 & 1.613 \\
\hline$\Delta n o_{t-1}$ & 0.107 & 0.079 & 0.125 & 0.081 & 0.140 \\
\hline$C A_{t-1}^{*}$ & 0.052 & 0.067 & 0.072 & 0.069 & 0.020 \\
\hline$r_{t-1}^{*}$ & - & -0.060 & 0.125 & -0.070 & 0.300 \\
\hline$\Delta n o_{t-2}$ & - & 0.067 & 0.016 & 0.066 & - \\
\hline$C A_{t-2}^{*}$ & - & 0.032 & 0.051 & 0.037 & - \\
\hline$r_{t-2}^{*}$ & - & -0.031 & 0.130 & -0.034 & - \\
\hline$x^{2}$ & 16.567 & 24.178 & 20.684 & 23.026 & 20.239 \\
\hline valor-p & 0.002 & 0.004 & 0.014 & 0.006 & 0.002 \\
\hline
\end{tabular}

Nota: $\gamma$ é a elasticidade de substituição intertemporal, e $a$ é a participação dos bens tradables no consumo final das famílias. Regressões correspondem ao período de 1991:1 a 2004:1.

Na Figura 3, são exibidos os valores da conta corrente provenientes dos dados e também as previsões geradas pela versão do modelo básico, painel (a). Com isso, se busca verificar se a análise gráfica pode ajudar a capturar a direção das flutuações na conta corrente.

Como se nota no painel (a) da Figura 3, o modelo simples da conta corrente, embora formalmente rejeitado, consegue determinar com bastante sucesso a direção geral das flutuações na conta corrente. Esse resultado vai na mesma direção da maioria dos testes realizados nessa área. Em geral, o modelo não satisfaz aos testes estatísticos, mas graficamente consegue determinar a direção das flutuações na conta corrente. Essa situação também ocorre no caso dos dados trimestrais brasileiros da década de 90 e do início dos anos 2000.

7 A ordem do VAR não altera significativamente o modelo. A estimativa do vetor $\mathbf{k}$, quando se usa um VAR de primeira ordem é $\mathbf{k}=\left[\begin{array}{ll}0,355 & -0,369\end{array}\right]$, distante do valor teórico [ $\left.\begin{array}{ll}0 & 1\end{array}\right]$. Para essa especificação a estatística $\chi^{2}$ com dois graus de liberdade corresponde a 7,42, com probabilidade de $2,45 \%$, o que leva a rejeitar o modelo do valor presente da conta corrente. 
Figura 3 - Valores reais e previstos da conta corrente - modelo básico e ampliado (1991:1-2004:1)
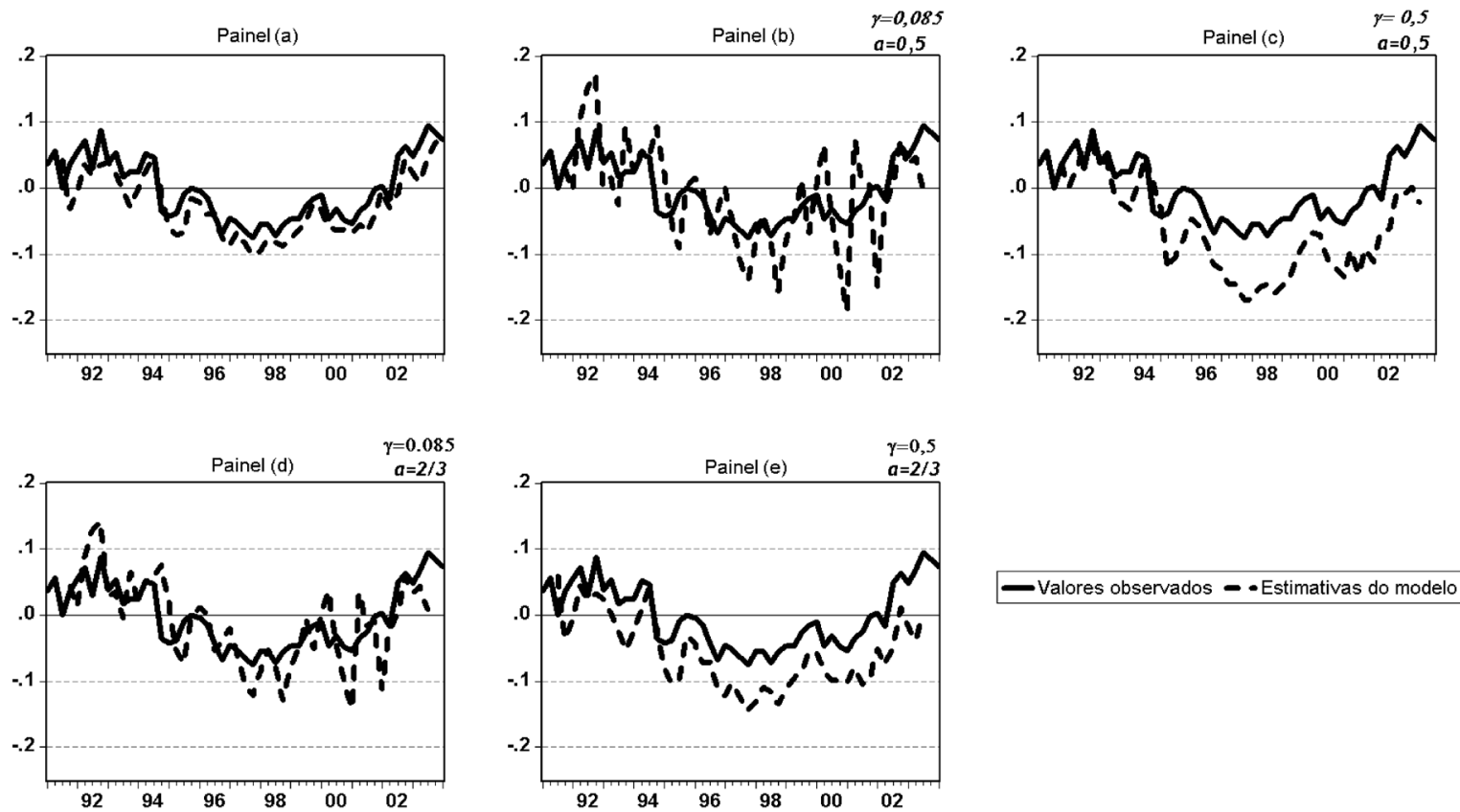

Direcionada a atenção para o modelo intertemporal com taxa de juros e câmbio variantes no tempo, percebe-se que ocorre apenas uma alteração marginal em relação ao modelo básico. Além disso, verifica-se que esse resultado independe dos valores utilizados para a elasticidade intertemporal e a participação dos bens tradables no consumo final privado. Como se pode ler na Tabela 2, o coeficiente sobre o valor atual da conta corrente, $C A_{t}^{*}$, é $-0,068$ para o caso em que $\gamma=0,085$ e $a=0,5$, sendo ligeiramente diferente para a combinação $\gamma=0,5$ e $a=0,5$, na qual o coeficiente sobre $C A^{*}{ }_{t}$ é -0,257. Quando $\gamma=0,5$ e $a=0,5$, o valor sobre $C A_{t}^{*}$ está um pouco mais distante de 1 e os valores sobre as demais varáveis, $\Delta n o_{t-i}, r_{t-i}^{*}(i=0,1,2)$ e $C A_{t-1}^{*}, C A_{t-2}^{*}$ afastam-se ainda mais dos valores esperados. Portanto, ainda que a diferença não seja significativa, quanto maior a elasticidade de substituição intertemporal, tanto maior o afastamento dos valores do vetor $\mathbf{k}$ em relação ao esperado. Essa mesma conclusão é verdadeira quando se altera o valor de $a$ para 0,67. Além disso, como confirmado no Apêndice B, para um dado valor de $\gamma$, a magnitude da participação dos bens comercializáveis no consumo final não gera alterações consideráveis no modelo.

Os painéis (b)-(e) da Figura 3 registram o insucesso da introdução das novas variáveis em melhorar o ajustamento do modelo. Cada gráfico representa o modelo para cada uma das combinações de $\gamma$ e $a$ que se está assumindo. Comparativamente ao gráfico do modelo básico, painel (a), a introdução de câmbio e juros piora o ajuste dos valores estimados em relação aos valores observados da conta corrente, tal qual definida. A combinação $\gamma=0,5$ e $a=2 / 3$ do modelo ampliado, painel (e), é a que melhor ajuda a capturar a trajetória da conta corrente. Nesse caso, o coeficiente de correlação entre as séries, observada e estimada, aproxima-se de 0,88 . Entretanto, do ponto de vista formal, o coeficiente sobre a taxa de juros $r_{t}{ }_{t}$ é consideravelmente diferente de zero. Portanto, a melhora em termos gráficos não necessariamente representa movimento em direção à aceitabilidade formal do modelo. Nos Apêndices D e F, nota-se que, para valores muito baixos de $\gamma$, a correlação entre as séries é baixa e as variâncias são estatisticamente diferentes. 
Portanto, os efeitos da taxa de câmbio real não se mostram suficientes no sentido de incrementar o modelo intertemporal da conta corrente. Isso parece um tanto surpreendente. De 1994 a 1999, a taxa a taxa de câmbio nominal foi utilizada pelos formuladores de política econômica como uma variávelchave no controle da inflação, o que implicou a apreciação da taxa de câmbio real. Essa apreciação da taxa de câmbio real, associada a fatores relacionados à abertura comercial, resultou em aumento crescente do déficit em transações correntes. Portanto, esperava-se que, com a introdução da taxa de câmbio real, o modelo conseguiria melhora na performance perante os testes formais. ${ }^{8}$

\subsection{Período 1947-2003}

O critério de informação AIC, não reportado, sugere que se estime um VAR de segunda ordem para o modelo básico; para o modelo ampliado, a ordem varia de 1 a 3, dependendo da escolha da elasticidade de substituição intertemporal e do coeficiente associado ao consumo de bens tradables. $\mathrm{Na}$ Figura 4, mostram-se os dados observados para a conta corrente, $C A^{*}$, e os valores estimados, $\left(C \hat{A}^{*}\right)$, derivados do modelo básico, painel (a), e ampliado, painéis (b) a (e).

Figura 4 - Valores reais e previstos da conta corrente - modelo básico e ampliado (anual)
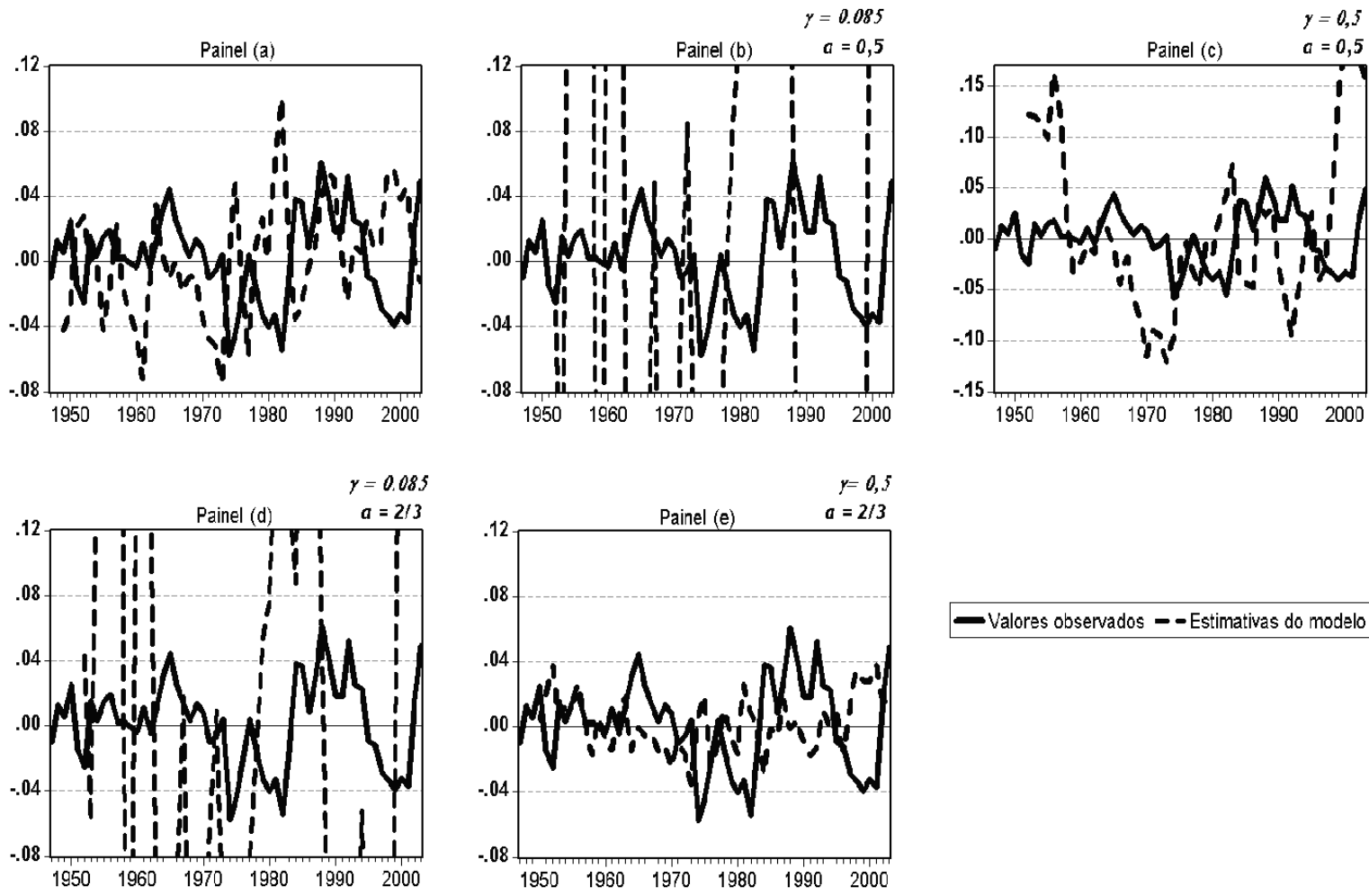

8 Choques nos mercados financeiros afetam significativamente a taxa de câmbio. Não há razão para supor correlação expressiva entre esses choques e os ajustes domésticos. Portanto, a introdução da taxa de câmbio real no modelo tende a gerar volatilidade excessiva. A definição da taxa de câmbio real utilizada neste artigo implica que todos os bens são comercializáveis e, assim, as definições de taxa de câmbio real interna e externa são equivalentes. Exploraram-se outras definições para a taxa de câmbio real, semelhantes às utilizadas por Araújo e Filho (2002). Os resultados encontrados, tanto para o período anual como para o período trimestral - não reportados, não indicaram alterações qualitativas significativas. O modelo de Iscan (2002) é uma alternativa para tratar essa questão de bens não comercializáveis. Por questões de delimitação e espaço, não exploramos essa possibilidade. Agradece-se a um parecerista anônimo desta revista por destacar esses pontos como possíveis explicações para o insucesso do modelo para a economia brasileira. 
Ao se tratar da amostra anual, graficamente os modelos básico e ampliado não possibilitam prever a direção correta da conta corrente. Em se tratando do modelo básico, nota-se que, em geral, os valores observados estão acima dos valores ótimos (estimados), o que pode sinalizar a existência de restrições na economia brasileira para a tomada de empréstimos no exterior. No que diz respeito ao modelo ampliado, percebe-se claramente o papel de $\gamma$ no ajuste. Os painéis (b) e (d), nos quais $\gamma$ $=0,085$, mostram que os valores estimados se afastam completamente dos valores realizados. Isso sugere que o fraco ajuste se deve aos valores escolhidos para $\gamma$ e não para $a$. Quando se faz $\gamma=0,5$, painéis (c) e (e), os valores estimados aproximam-se dos dados da conta corrente. Graficamente, o melhor ajuste ocorre quando se tem $\gamma=0,5$ e $a=2 / 3$, painel (e).

As séries estimadas tomando $\gamma=0,5$, painéis (c) e (e), confirmam o resultado gerado pelo modelo básico em relação à mobilidade de capitais. Por exemplo, observando o gráfico do painel (e), verifica-se nos anos 80 a escassez de recursos externos para financiar o volume de consumo desejado pelos indivíduos. Essa conclusão é válida na medida em que a série ótima, $C \hat{A}^{*}$, possui nível menor do que os valores realizados da série de conta corrente da economia brasileira. Entretanto, a partir da segunda metade da década de 90 , os valores de $C \hat{A}^{*}$ estão bastante acima da série da conta corrente, o que pode sugerir a presença de maiores fluxos de capitais de curto prazo decorrentes de movimentos especulativos.

O ajuste do modelo para descrever a trajetória do saldo em transações correntes reflete-se nos testes estatísticos apresentados na Tabela 3. Os resultados apresentados nessa tabela descrevem novamente o modelo básico e o ampliado. Neste último, para análise de sensibilidade, seguiu-se um procedimento similar a Bergin e Sheffrin (2000) e utilizaram-se dois valores diferentes de $a$ e $\gamma$.

Tabela 3 - Teste do modelo intertemporal da conta corrente - anual

\begin{tabular}{|c|c|c|c|c|c|}
\hline \multirow{3}{*}{ k' } & \multirow{3}{*}{ Modelo básico } & \multicolumn{4}{|c|}{ Modelo ampliado } \\
\hline & & \multicolumn{2}{|c|}{$a=1 / 2$} & \multicolumn{2}{|c|}{$a=2 / 3$} \\
\hline & & $\gamma=0,085$ & $\gamma=0,5$ & $\gamma=0,085$ & $\gamma=0,5$ \\
\hline$\Delta n o_{t}$ & -0.503 & -0.872 & -0.691 & -0.789 & -0.341 \\
\hline$C A_{t}^{*}$ & 0.523 & 0.635 & 0.653 & 0.347 & 0.734 \\
\hline$r_{t}^{*}$ & - & -0.013 & 0.236 & -0.031 & 0.581 \\
\hline$\Delta n o_{t-1}$ & -0.165 & -0.433 & -0.275 & -0.383 & - \\
\hline$C A_{t-1}^{*}$ & -0.162 & 0.238 & 0.285 & 0.127 & - \\
\hline$r_{t-1}^{*}$ & - & -0.077 & -0.298 & -0.084 & - \\
\hline$\Delta n o_{t-2}$ & - & -0.227 & -0.080 & -0.229 & - \\
\hline$C A_{t-2}^{*}$ & - & 0.112 & 0.190 & -0.065 & - \\
\hline$r_{t-2}^{*}$ & - & 0.012 & 0.160 & 0.003 & - \\
\hline$\chi^{2}$ & 6.495 & 18.772 & 9,959 & 14.880 & 6.032 \\
\hline valor-p & 0.165 & 0.005 & 0.354 & 0.094 & 0.110 \\
\hline
\end{tabular}

Nota: $\gamma$ é a elasticidade de substituição intertemporal, e $a$ é a participação dos bens tradables no consumo final das famílias. Regressões correspondem ao período de 1947 a 2003.

Para o modelo básico, o valor do elemento do vetor $\mathbf{k}$ associado à conta corrente é 0,523 , razoavelmente próximo do valor teórico de 1 . O mesmo acontece com os coeficientes associados ao produto líquido e à defasagem da conta corrente. A proximidade desses coeficientes dos valores te- 
óricos reflete-se na estatística $\chi^{2}(4)$ do teste Wald das restrições do modelo, equação (42). Conforme se nota pelo p-valor, 0,165, não é possível rejeitar a hipótese nula de que o vetor $\mathrm{k}$ como um todo seja $\left[\begin{array}{llll}0 & 1 & 0 & 0\end{array}\right]$ nem ao menos ao nível de $10 \%$.

Como se pode observar na Tabela 3, o modelo revela resultados contraditórios quando inseridas a taxa de juros e a de câmbio real. O modelo revela certa sensibilidade em relação à escolha da elasticidade de substituição intertemporal. Para o caso em que $\gamma=0,085$ e $a=0,5$, o coeficiente sobre $C A^{*}{ }_{t}^{*}$ passa a ser 0,635 , gerando melhora comparativamente ao coeficiente do modelo básico; contudo, os coeficientes associados ao produto líquido afastam-se de seus valores esperados. Embora a estatística $\chi^{2}(9)$ não possa ser comparada ao caso anterior em virtude do aumento do número de graus de liberdade, a comparação com o valor-p é válida. Note-se que o valor-p diminui para 0,005, o que permite rejeitar a hipótese nula das restrições impostas pelo modelo ao nível de $5 \%$. Essa piora, em menor grau, permanece quando mantido o mesmo $\gamma$ e aumentada a participação dos bens tradables, fazendo $a=2 / 3$. Entretanto, para o último caso, só se pode rejeitar o modelo em nível mais elevado.

No entanto, quando se toma $\gamma=0,5$, nenhuma novidade é gerada no sentido da não-rejeição do modelo comparativamente ao caso básico. A combinação de $\gamma=0,5$ e $a=0,5$ aumenta inclusive o valor-p. Quando se aumenta $a$ para 2/3, dado $\gamma=0,5$, o valor-p diminui sensivelmente, não a ponto de provocar a rejeição do modelo nem ao menos no nível de $10 \%$. Portanto, nota-se que, para o período 1947-2003, o modelo é mais sensível à $\gamma$, de tal forma que valores diferentes desse parâmetro permitem que os testes das restrições impostas pelo modelo levem a conclusões distintas em relação ao modelo intertemporal da conta corrente com câmbio e juros.

Em decorrência desse resultado, parece valioso ampliar os valores de $\gamma$, tal como mostrado na Figura 5 e no Apêndice A. Note-se no apêndice que o valor máximo de $\gamma$ no qual as restrições do modelo são atendidas independe da medida de $a$. Todavia, existe sensibilidade em relação ao limite inferior. Quanto menor $a$ tanto menor $\gamma$, o que equivale dizer que a região de $\gamma$, na qual o modelo não é rejeitado varia inversamente ao valor de $a$. $\operatorname{Com} a=1 / 2$, temos $0,225<\gamma<1,145$; se $a=1$ então $0,425<\gamma<1,135$ no nível de 5\%. Essa constatação ressalta a importância de se inserirem bens não-comercializáveis no modelo, pois, se $a=1$, então o modelo contempla somente juros e todos os bens são comercializáveis.

Na Figura 5, revela-se a região de valores de $\gamma$ na qual o modelo ampliado com juros e câmbio atende às restrições a ele impostas em um nível de significância de 5\%. A Figura 5 mostra que, para $0,22<\gamma<1,15$, implica valor-p maior que 0,05 ; as restrições são, pois, atendidas. ${ }^{9}$ O importante da discussão é destacar que o modelo pode ser compatível tanto quanto os valores de $\gamma$ proposto por Reis et al. (1998) bem como por Issler e Piqueira (2000), tal como comentado na Seção 4.

9 Note-se que existe ligeira diferença entre os valores mostrados na figura e os da Tabela 4. Essa discrepância se explica pelo fato que foi usado, por simplicidade computacional, um VAR(3) para todos os valores da elasticidade de substituição intertemporal na construção da Figura 5. A conclusão qualitativa não é alterada por essa simplificação. 
Figura 5 - Valores da elasticidade de substituição intertemporal compatíveis com o modelo

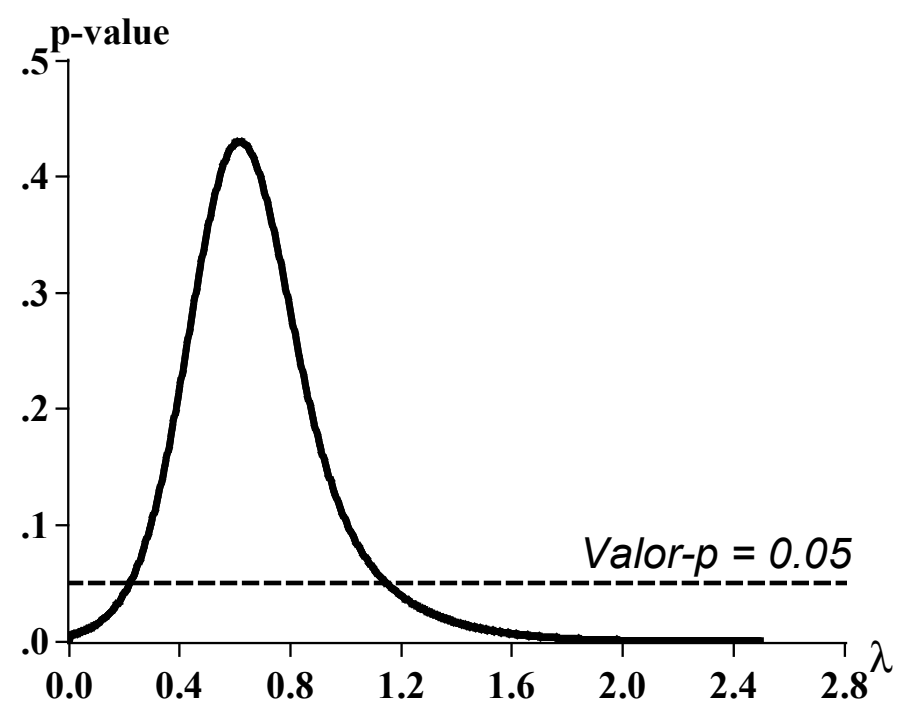

Nota: $\gamma$ é a elasticidade de substituição intertemporal e valor-p indica a probabilidade de se obter uma estatística de teste cujo valor absoluto é maior ou igual ao valor da estatística amostral se a hipótese nula de que os coeficientes sejam iguais ao vetor $\mathbf{k}=\left[\begin{array}{lllllll}0 & 1 & 0 & 0 & 0 & 0 & \ldots\end{array}\right]$ é verdadeira. A participação dos bens tradables no consumo final é da ordem de $50 \%, a=0,5$. Para todas os valores de $\gamma$, estimou-se um var(3).

No sentido de se verificar a sensibilidade do modelo em relação à taxa de juros, à taxa de câmbio e à elasticidade de substituição intertemporal, usaram-se diversas combinações de valores e especificações do modelo. Para todos os casos apresentados, a hipótese nula testada é de que as restrições impostas pelo modelo teórico são atendidas. Os testes podem ser analisados por meio do valor-p.

Analisados os testes com base no exposto acima, verifica-se que a teoria não pode ser rejeitada quando usado o modelo básico, tal como dito. O valor-p encontrado é de 0.165 ; portanto, não se pode rejeitar a hipótese nula sobre as restrições do modelo. Os coeficientes sobre $\Delta n o_{t-i}, C A_{t-i}^{*}, r_{t-i}^{*}$ $(i=0,1,2)$ estão próximos dos valores teóricos.

Quando se move para um modelo em que novas variáveis são introduzidas encontram-se resultados em direções opostas. Quando se trabalha somente com a taxa de juros, o modelo não é rejeitado apenas quando utilizados valores baixos para a elasticidade de substituição intertemporal. Ao se tomar $\gamma=0.085$ e 0.333 , tem-se que os valores-p são 0.183 e 0.123 , respectivamente. Esses valores não possibilitam rejeitar a hipótese nula de que os dados geram valores consistentes com as restrições impostas pelo modelo teórico. Novamente, os valores sobre $\Delta n o_{t-i}, C A_{t-i}^{*}, r_{t-\mathrm{i}}^{*}$ são razoavelmente próximos dos valores esperados. 
Tabela 4 - Análise de sensibilidade

\begin{tabular}{|c|c|c|c|c|c|c|c|c|c|c|c|c|}
\hline Modelo & $\gamma$ & $\Delta n o_{t}$ & $C A_{t}^{*}$ & $r_{t}^{*}$ & $\Delta n o_{t-1}$ & $C A_{t-1}^{*}$ & $r_{t-1}^{*}$ & $\Delta n o_{t-2}$ & $C A_{t-2}^{*}$ & $r_{t-2}^{*}$ & $x^{2}$ & Valor- $p$ \\
\hline Teórico & - & 0.00 & 1.00 & 0.00 & 0.00 & 0.00 & 0.00 & 0.00 & 0.00 & 0.00 & - & - \\
\hline \multirow[t]{3}{*}{ Básico } & - & -0.50 & 0.52 & - & -0.16 & -0.16 & - & - & - & - & 6.50 & 0.165 \\
\hline & 0.085 & -0.45 & 0.53 & 0.59 & -0.13 & -0.21 & 0.50 & - & - & - & 8.84 & 0.183 \\
\hline & 0.333 & -0.33 & 0.49 & 1.55 & -0.11 & -0.33 & 0.84 & - & - & - & 10.05 & 0.123 \\
\hline \multirow[t]{5}{*}{ A } & 0.500 & -0.26 & 0.46 & 2.20 & -0.10 & -0.40 & 1.07 & - & - & - & 11.48 & 0.075 \\
\hline & 0.800 & -0.13 & 0.41 & 3.36 & -0.07 & -0.53 & 1.49 & - & - & - & 15.07 & 0.020 \\
\hline & 1.500 & 0.19 & 0.29 & 6.08 & -0.01 & -0.85 & 2.46 & - & - & - & 26.02 & 0.000 \\
\hline & 0.085 & -0.87 & 0.65 & -0.01 & -0.44 & 0.22 & -0.08 & -0.24 & 0.10 & 0.01 & 21.68 & 0.010 \\
\hline & 0.333 & -0.80 & 0.41 & -0.12 & -0.40 & 0.13 & -0.33 & -0.24 & -0.04 & 0.01 & 18.60 & 0.029 \\
\hline \multirow[t]{5}{*}{ B } & 0.500 & -0.76 & 0.25 & -0.29 & -0.37 & 0.06 & -0.54 & -0.24 & -0.14 & -0.04 & 13.76 & 0.131 \\
\hline & 0.800 & -0.67 & -0.04 & -1.53 & -0.32 & -0.05 & -1.24 & -0.24 & -0.31 & -0.59 & 10.85 & 0.286 \\
\hline & 1.500 & -0.48 & -0.72 & 1.80 & -0.20 & -0.31 & -0.66 & -0.24 & -0.72 & 1.24 & 12.62 & 0.181 \\
\hline & 0.085 & -0.87 & 0.64 & -0.01 & -0.43 & 0.24 & -0.08 & -0.23 & 0.11 & 0.01 & 21.04 & 0.013 \\
\hline & 0.333 & -0.79 & 0.52 & 0.00 & -0.36 & 0.24 & -0.28 & -0.17 & 0.08 & 0.07 & 13.66 & 0.135 \\
\hline \multirow[t]{3}{*}{$C$} & 0.500 & -0.69 & 0.65 & 0.24 & -0.27 & 0.28 & -0.30 & -0.08 & 0.19 & 0.16 & 9.96 & 0.354 \\
\hline & 0.800 & -0.20 & 0.73 & 1.50 & - & - & - & - & - & - & 8.02 & 0.046 \\
\hline & 1.500 & 0.02 & -0.92 & 4.22 & -0.22 & -1.14 & 0.47 & - & - & - & 26.04 & 0.000 \\
\hline
\end{tabular}

Nota: A corresponde ao modelo que inclui somente a taxa de juros; $\mathbf{B}$ somente a taxa de câmbio e $\mathbf{C}$ o modelo ampliado com juros e câmbio. $\chi^{2}$ é a estatística do teste Wald sob a hipótese nula de que os coeficientes sejam iguais ao vetor $\mathbf{k}=\left[\begin{array}{ll}0 & 10\end{array}\right.$ $\left.\begin{array}{llllllll}0 & 0 & 0 & \ldots & 0\end{array}\right]$.

Porém, ainda usando apenas a taxa de juros real, a situação inverte-se para valores maiores da elasticidade de substituição intertemporal. Percebe-se claramente que quanto maior o valor de $\gamma$ tanto maior a probabilidade de rejeitar a hipótese nula. Como os valores dos coeficientes do VAR não são alterados, ${ }^{10}$ estimativas elevadas de $\gamma$ representam piora das restrições no teste Wald. Por exemplo, observando a equação (42) percebe-se que deve ter satisfeita a condição $a_{21}-a_{11}+\gamma a_{31}=0$. Claramente, uma vez que essa restrição tenha sido atendida para valores baixos da elasticidade de substituição, quanto maior o valor de $\gamma$ menor a possibilidade de se atender a essa restrição, dado que os valores de $a_{2 j}$ não variam no caso em que se utiliza apenas a taxa de juros real. A mesma observação vale para as demais restrições da equação (42). Note-se também a piora dos coeficientes associados a $\Delta n o_{t-i}, C A_{t-i}^{*}, r_{t-i}^{*}$, principalmente sobre $r^{*}$.

Para uma situação em que é mantida a taxa de juros constante e permitidas variações da taxa de câmbio real, nota-se que as restrições são atendidas para determinada região de valores de $\gamma$. Para essa especificação, a elasticidade afeta o modelo por dois canais. Em primeiro lugar, valores diferentes de $\gamma$ alteram a série $\left\{r^{*}\right\}$, conforme equação (32) e conseqüentemente os valores dos coeficientes do VAR. Além disso, como no caso anterior, $\gamma$ também altera as restrições testadas, equação (42). Como se observa na Tabela 4, rejeita-se formalmente o modelo, com câmbio apenas para valores baixos da elasticidade de substituição intertemporal.

10 Note-se, por meio de (32), que se é imposto $\Delta p=0$, mudanças em $\gamma$ não alteram $r^{*}$; portanto, a estimativa do VAR (35) independe de $\gamma$. 


\subsection{Variância e correlação}

Como salienta Ghosh (1995), aproveitando-se do fato que já se calculou a conta corrente ótima, pode-se compará-la aos valores observados das transações correntes no sentido de analisar a volatilidade das séries. Ainda, também se pode calcular a correlação entre as séries e verificar se os movimentos da conta corrente são consistentes com as hipóteses do modelo teórico.

Na Tabela 5, compararam-se os desvios padrão e as correlações entre a série real da conta corrente e as séries estimadas, obtidas das várias especificações usadas neste trabalho. Dos Apêndices $\mathrm{C}$ a F, foram construídos gráficos para uma região contínua de $\gamma$. Aqui, para comparação, foram utilizadas as mesmas combinações de elasticidade de substituição intertemporal e share dos bens comercializáveis, na qual se construíram os gráficos anteriores. Como resultado importante, ao nível de significância de 5\%, não se rejeitou a hipótese nula de que as variâncias são iguais entre as séries reais da conta corrente e as séries construídas pelo modelo básico, para ambos os períodos.

Em direção oposta, as variâncias das séries obtidas pelo modelo ampliado são, em quase todas as especificações e os períodos, estatisticamente diferentes das séries reais da conta corrente. Portanto, a inclusão da taxa de juros e da taxa de câmbio no modelo tem considerável impacto nos resultados, porém em sentido contrário. Em quase todos os casos, o modelo ampliado aumenta o desvio padrão da série ótima. ${ }^{11}$ A maior volatilidade de uma série estimada implica que a mobilidade de capitais no Brasil não é perfeita, existindo restrições para que os agentes otimizem o consumo via conta corrente.

Tabela 5 - Variância e correlação entre as séries - estimada e real

\begin{tabular}{lcccccc}
\hline & Real & Básico & \multicolumn{5}{c}{ Juros e Câmbio } \\
\cline { 5 - 6 } & & & $\mathbf{A}$ & $\mathbf{B}$ & $\mathbf{C}$ & $\mathbf{D}$ \\
\hline 1991:1-2004:1 & & & & & & \\
$\sigma$ & 0.049 & 0.0471 & 0.0772 & 0.0685 & 0.0650 & 0.0539 \\
Razão $\left(\sigma_{\mathrm{CA}} / \sigma_{\mathrm{CA}^{*}}\right)$ & - & 1.0398 & 0.6343 & 0.7150 & 0.7537 & 0.9085 \\
Teste-F & - & 1.0811 & 2.6400 & 2.0780 & 1.8698 & 1.3139 \\
Valor-p & - & 0.7839 & 0.0012 & 0.0136 & 0.0343 & 0.3475 \\
Correlação & - & 0.9022 & 0.6198 & 0.8367 & 0.7154 & 0.8393 \\
1947-2003 & & & & & & \\
$\sigma$ & 0.028 & 0.0356 & 0.6365 & 0.0833 & 0.3849 & 0.0172 \\
Razão $\left(\sigma_{\mathrm{CA}} / \sigma_{\mathrm{CA}}\right)$ & - & 0.7832 & 0.0439 & 0.3353 & 0.0725 & 1.6252 \\
Teste-F & - & 1.6301 & 500.5922 & 8.5670 & 183.0873 & 2.6905 \\
Valor-p & - & 0.0753 & 0.0000 & 0.0000 & 0.0000 & 0.0004 \\
Correlação & - & -0.3749 & 0.0923 & -0.0959 & 0.0788 & -0.3183 \\
\hline
\end{tabular}

Nota: $\operatorname{Em~A:~}(\gamma ; a)=(0.085 ; 0.500) ; \mathbf{B}:(\gamma ; a)=(0.5 ; 0.500) ; \mathbf{C}:(\gamma ; a)=(0.085 ; 0.67) ; \mathbf{D}:(\gamma ; a)=(0.5 ; 0.67) . \sigma$ é o desvio-padrão e razão é a divisão entre o desvio-padrão da série da conta corrente real pelo desvio-padrão das séries estimadas. Teste-F é o teste F para a igualdade da variância entre as séries, para a hipótese nula de que são iguais. Correlação é o coeficiente de correlação entre a série real e a respectiva série estimada dentro da amostra.

11 No Apêndice C existe pequena região de valores de $\gamma$. próximos de 0,8 , que produzem igualdade entre as variâncias para o período 1947-2003. No caso 1991:1-2004:1, essa região é maior e situa-se entre 0,2 e 0,5, como se pode ver no Apêndice D. 
Em relação à correlação, tem-se algo muito similar ao caso da variância quando observado o período 1991:1-2004:1. Qualquer que seja a especificação do modelo ampliado, ela diminui, comparativamente ao modelo básico. Entretanto, de acordo com o Apêndice F, as especificações do modelo conseguem explicar consideravelmente bem a direção dos movimentos da conta corrente, desde que o valor de $\gamma$ não seja muito baixo. Já no período 1947-2003, a correlação entre as séries estimadas e a série real da conta corrente é fraca, mesmo para o modelo básico. Ao se observar o Apêndice E, constata-se que o coeficiente de correlação vai piorando conforme se aumenta o valor de $\gamma$, inclusive tornando-se negativo a partir do momento em que $\gamma$ é superior a 0,3 , independentemente do valor de $a$.

\section{CONCLUSÃO}

Neste artigo foi apresentado e testado com dados da economia brasileira o modelo intertemporal da conta corrente desenvolvido por Bergin e Sheffrin (2000), no qual a taxa de juros mundial e a taxa de câmbio real são variantes no tempo. A inclusão dessas variáveis no modelo básico significa considerar novos efeitos sobre a economia. Com a taxa de juros variável no tempo, os agentes podem substituir consumo presente por consumo futuro quando a taxa de juros estiver acima de seu nível normal. Esse efeito ocorre na medida em que tomar empréstimos se torna mais dispendioso. Em relação à taxa de câmbio, variações nessa variável acarretam substituições intertemporais e intratemporais.

Levados em conta os dados disponíveis, consideram-se dois períodos para efetuar os testes. A amostra trimestral abarcou do primeiro trimestre de 1991 ao primeiro trimestre de 2004. O período anual compreendeu de 1947 a 2003. Consideraram-se basicamente três variáveis. A primeira foi o produto líquido, definido como o Produto Interno Bruto descontado do consumo final da Administração Pública e da formação bruta de capital. A conta corrente foi a segunda variável usada. Esta foi definida como o produto líquido descontado do consumo final das famílias. A terceira variável correspondeu à combinação da taxa de juros mundial com a taxa de câmbio real. Todas as variáveis se revelaram estacionárias, exceto a conta corrente para o período 1991:1-2004:1.

De forma geral, os testes levam a rejeitar o modelo intertemporal de Bergin e Sheffrin (2000) para o Brasil. Em relação ao período trimestral, a rejeição independe dos parâmetros utilizados. Já no caso anual, o modelo é rejeitado quando usados valores baixos para a elasticidade de substituição intertemporal. Ao se levar em conta a observação de Hall (1988) de que a elasticidade de substituição intertemporal deve situar-se abaixo de 0,1 , visto que o consumo tende a responder fracamente à taxa de juros real, pode-se rejeitar o modelo para explicar o comportamento da conta corrente no Brasil. Contudo, quando se considera a estimativa de Issler e Piqueira (2000), o modelo não pode ser rejeitado ao se levar em conta a restrição testada nesse artigo.

Para verificar se o modelo ampliado segue o padrão do modelo simples, qual seja, em certos casos rejeição formal e adequação gráfica, elaboraram-se gráficos nos quais foram inseridos os valores observados e previstos da conta corrente. No período 1947-2003, verificou-se que os resultados não são consistentes: mesmo com diferentes valores da elasticidade de substituição intertemporal, o coeficiente de correlação entre as séries é baixo e, em alguns casos, negativo. Portanto, o modelo não consegue apontar a direção das flutuações da conta corrente quando olhado o horizonte 19472003. Entretanto, para o período trimestral, 1991:1-2004:1, o coeficiente de correlação mostrou-se 
bastante alto para todas as especificações do modelo em que a elasticidade de substituição não fosse extremamente baixa.

Pelas séries ótimas construídas para a conta corrente, que deveriam ser os valores observados dados os choques na economia, pode-se estabelecer variâncias, tomadas como benchmark, para compará-las com os valores reais. Enquanto o modelo básico indica que a conta corrente é mais volátil do que seria caso os agentes estivessem comportando-se otimamente, o modelo ampliado, em geral, aponta em direção contrária, ou seja, a volatilidade real da conta corrente é menor do que a série teórica. Isso revela que o modelo básico prediz excesso de mobilidade de capital, enquanto o modelo ampliado anuncia que a economia brasileira apresenta restrições no fluxo de capitais internacionais, o que equivale a dizer que existem dificuldades para a alocação intertemporal do consumo diante dos choques no produto líquido, na taxa de juros e na taxa de câmbio real.

As conclusões acima sugerem o emprego de esforço adicional de pesquisa para se enriquecer o entendimento da dinâmica das transações correntes no Brasil. Menciona-se a presença de três suposições fortes do modelo tratado aqui: a separação das decisões de investimento e poupança; a ausência de relação entre investimento e consumo final da administração pública e a inexistência de distorções no mercado de capitais. O abrandamento dessas hipóteses em trabalhos realizados futuramente pode trazer consigo considerável melhora nos resultados por algumas razões. Talvez a mais importante delas esteja ligada ao fato de que imperfeições no mercado de capitais tendem a provocar influência da poupança nacional e do consumo do governo sobre o investimento. Em tal ambiente de distorção, variações nos gastos do governo, por exemplo, podem alterar a taxa de juros e, assim, causar impacto no investimento doméstico via efeito deslocamento - crowding out. 


\section{APÊNDICE}

\section{APÊNDICE A - VALORES DE $\gamma$ COMPATÍVEIS COM O MODELO (ANUAL: 1947-2003)}

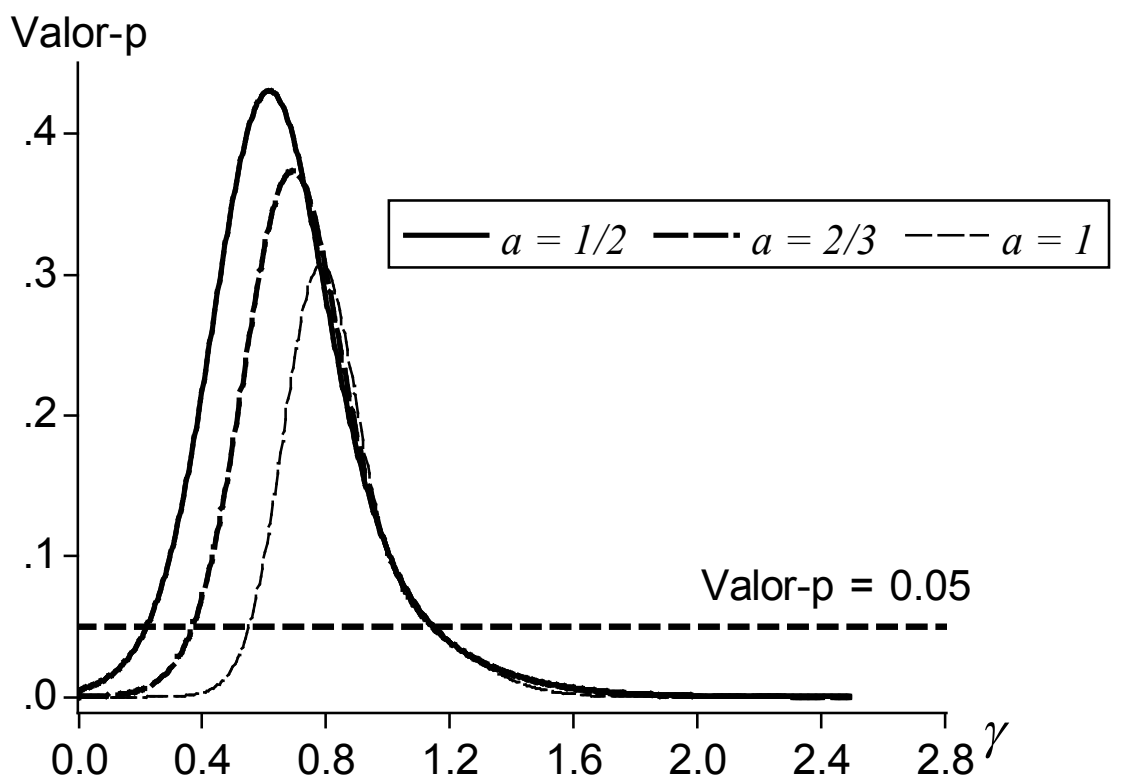

Nota: $\gamma$ é a elasticidade de substituição intertemporal e valor-p indica a probabilidade de se obter uma estatística de teste cujo valor absoluto é maior ou igual ao valor da estatística amostral se a hipótese nula de que os coeficientes sejam iguais ao vetor $\mathbf{k}=\left[\begin{array}{lllllll}0 & 1 & 0 & 0 & 0 & 0 & \ldots\end{array}\right]$ é verdadeira. $a$ é a participação dos bens tradables no consumo final. Para todas os valores de $\gamma$ estimou-se um var(3). 


\section{APÊNDICE B - VALORES DE $\gamma$ COMPATÍVEIS COM O MODELO (TRIMESTRAL: 1991:1- 2004:1)}

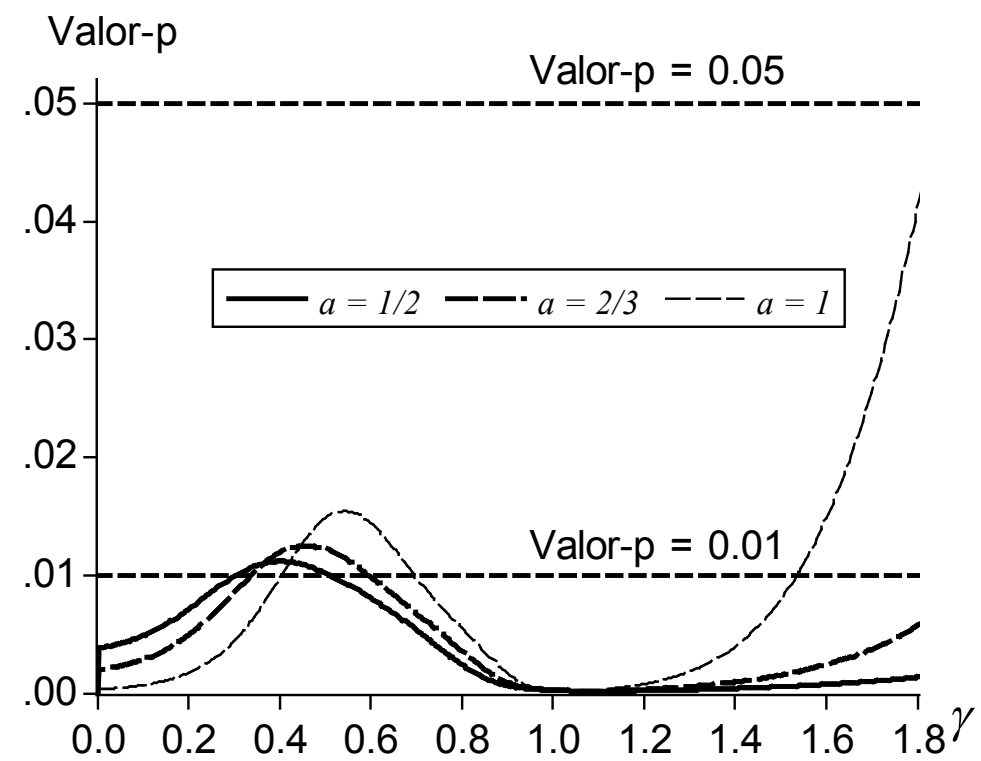

Nota: $\gamma$ é a elasticidade de substituição intertemporal, e valor-p indica a probabilidade de se obter uma estatística de teste cujo valor absoluto é maior ou igual ao valor da estatística amostral se a hipótese nula de que os coeficientes sejam iguais ao vetor $\mathbf{k}=\left[\begin{array}{lllllll}0 & 1 & 0 & 0 & 0 & 0 & \ldots\end{array}\right]$ é verdadeira. $a$ é a participação dos bens tradables no consumo final. Para todas os valores de $\gamma$, estimou-se um var(3).

\section{APÊNDICE C - TESTE DA IGUALDADE ENTRE VARIÂNCIAS (ANUAL: 1947-2003)}
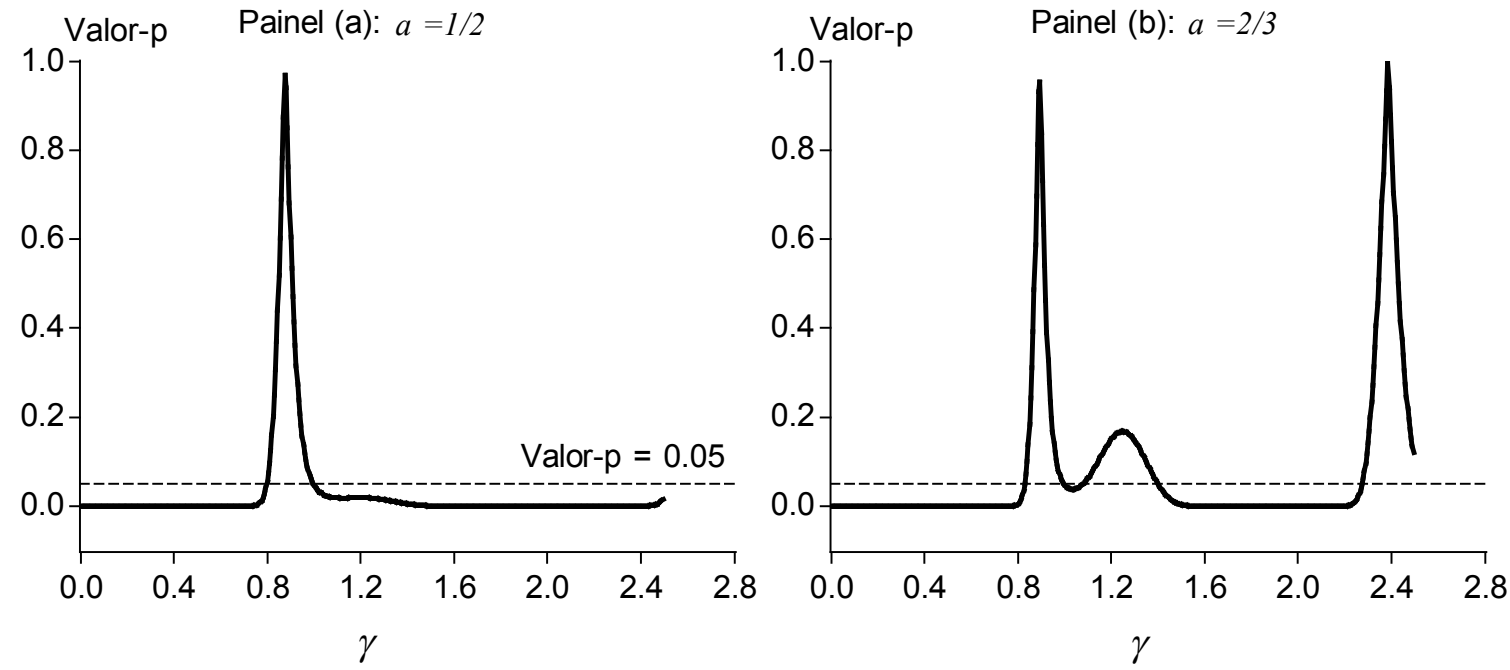

Nota: $\gamma$ é a elasticidade de substituição intertemporal; $a$ é participação dos bens tradables no consumo final e valor-p indica a probabilidade de se obter uma estatística de teste cujas variâncias das séries real e estimada da conta corrente sejam iguais. 
APÊNDICE D - TESTE DA IGUALDADE ENTRE VARIÂNCIAS (TRIMESTRAL: 1991:12004:1)
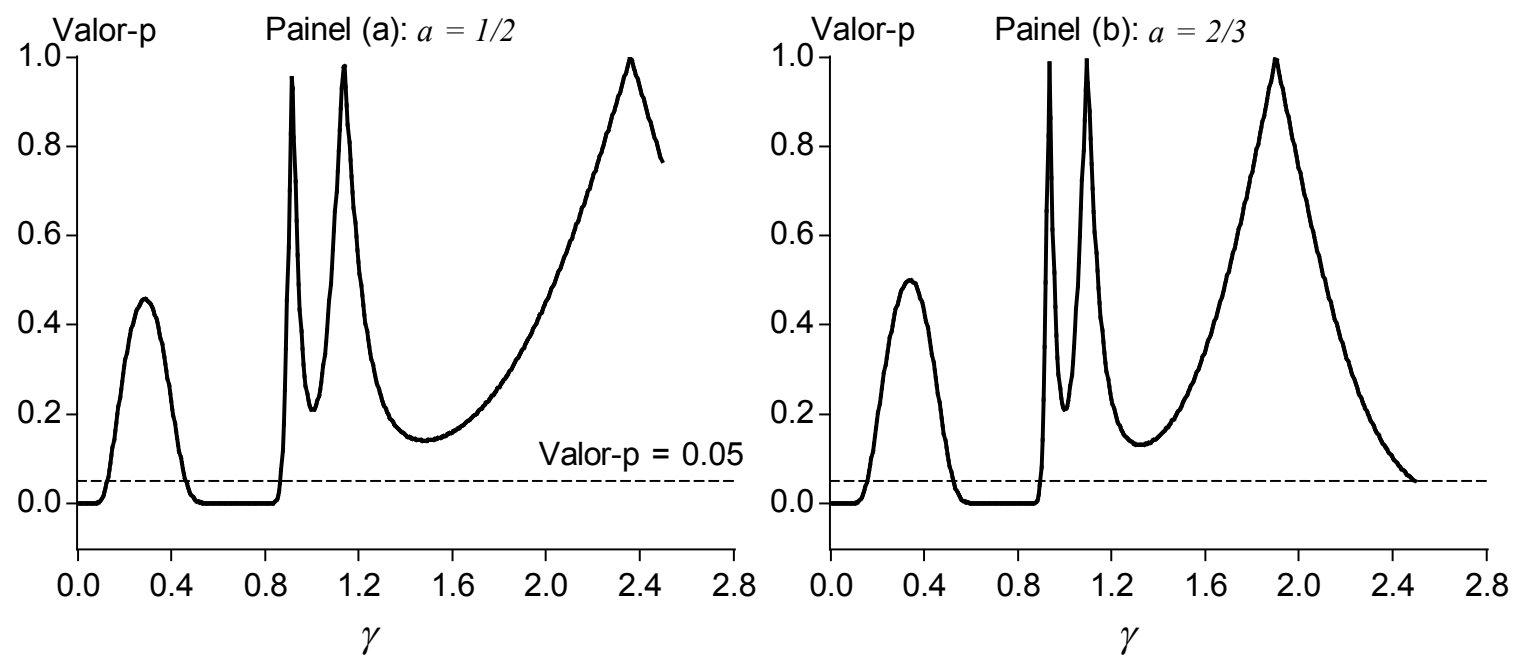

Nota: $\gamma$ é a elasticidade de substituição intertemporal; $a$ é participação dos bens tradables no consumo final e valor-p indica a probabilidade de se obter uma estatística de teste cujas variâncias das séries real e estimada da conta corrente sejam iguais.

APÊNDICE E - CORRELAÇÃo (ANUAL: 1947-2003)

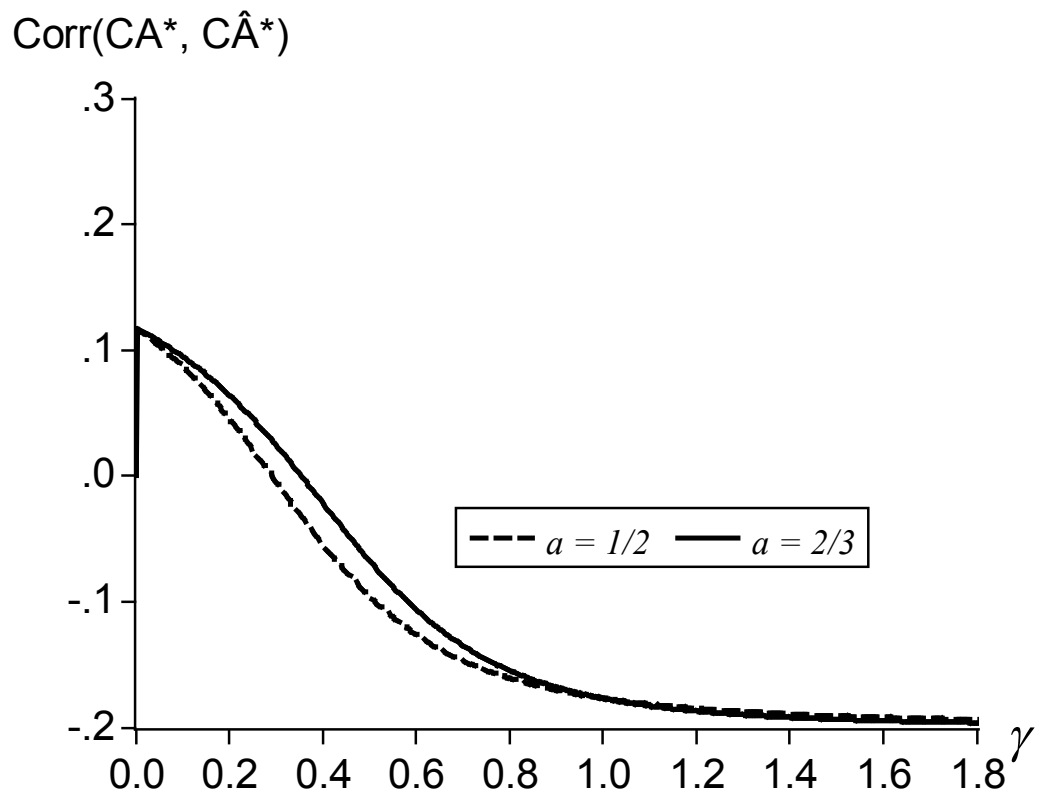

Nota: $C A^{*}$ é a série observada da conta corrente, e $C \hat{A}^{*}$ é a respectiva estimativa calculada de acordo com (39). 


\section{APÊNDICE F - CORRELAÇÃO (TRIMESTRAL: 1991:1 - 2004:1)}

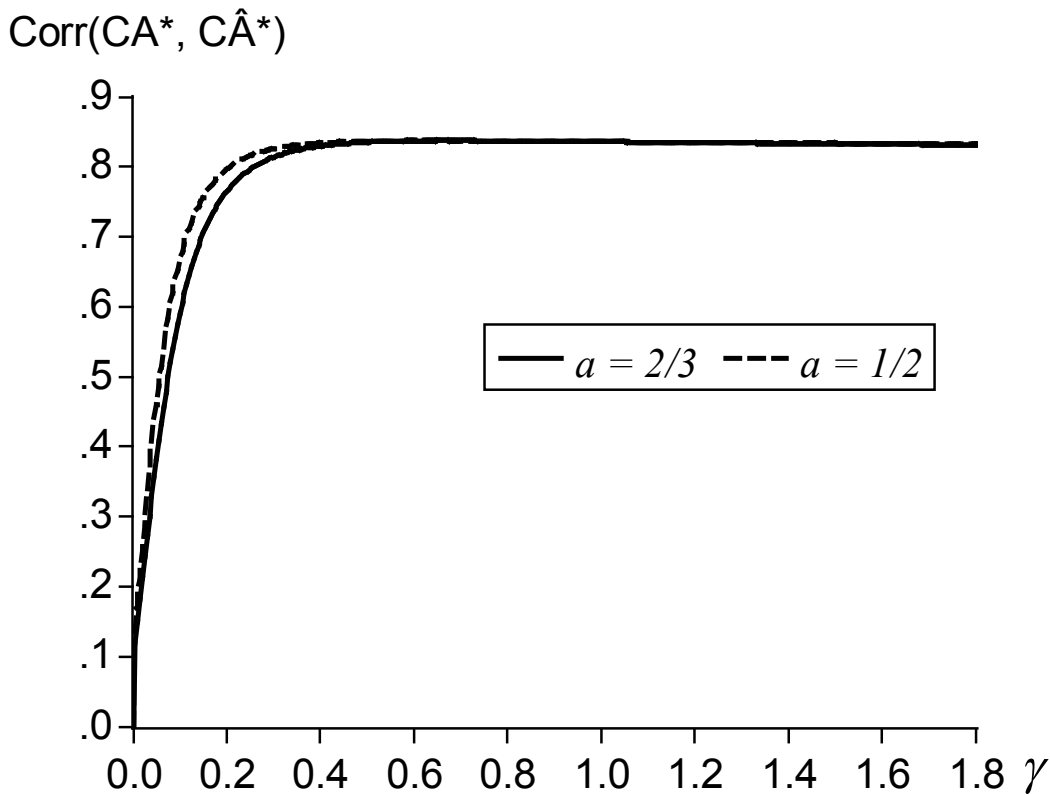

Nota: $C A^{*}$ é a série observada da conta corrente, e $C \hat{A}^{*}$ é a respectiva estimativa calculada de acordo com (39).

\section{APÊNDICE G - ELASTICIDADES}

A condição de Euler do problema de maximização é vista como:

$$
\left(\frac{P_{t}^{*}}{P_{t+1}^{*}}\right)\left(\frac{C_{t}^{*}}{C_{t+1}^{*}}\right)=\left[\beta\left(1+r_{t+1}\right)\right]^{-1 / \sigma}\left(\frac{P_{t}^{*}}{P_{t+1}^{*}}\right)^{\frac{\sigma-1}{\sigma}}
$$

Elasticidade do gasto em relação à mudança no preço relativo:

$$
\frac{\partial\left(C_{t} / C_{t+1}\right)}{\partial\left(P_{t}^{*} / P_{t+1}^{*}\right)} \frac{\left(P_{t}^{*} / P_{t+1}^{*}\right)}{\left(C_{t} / C_{t+1}\right)}=(1-\gamma)(1-a)
$$

Elasticidade do gasto em consumo em relação à taxa de juros:

$$
\frac{\partial\left(C_{t} / C_{t+1}\right)}{\partial\left(1+r_{t+1}\right)} \frac{\left(1+r_{t+1}\right)}{\left(C_{t} / C_{t+1}\right)}=\gamma
$$


Elasticidade do gasto em relação à mudança nos preços:

$$
\frac{\partial\left(C_{t} / C_{t+1}\right)}{\partial\left(P_{t} / P_{t+1}\right)} \frac{\left(P_{t} / P_{t+1}\right)}{\left(C_{t} / C_{t+1}\right)}=\left[\frac{\partial\left(C_{t} / C_{t+1}\right)}{\partial\left(P_{t}^{*} / P_{t+1}^{*}\right)} \frac{d\left(P_{t}^{*} / P_{t+1}^{*}\right)}{d\left(P_{t} / P_{t+1}\right)}\right] \frac{\left(P_{t} / P_{t+1}\right)}{\left(C_{t} / C_{t+1}\right)}=(1-\gamma)(1-a)
$$

Elasticidade da cesta em relação à $\left(P_{t} / P_{t+1}\right)$ - efeito intertemporal:

$$
\frac{\partial\left(C_{t}^{*} / C_{t+1}^{*}\right)}{\partial\left(P_{t} / P_{t+1}\right)} \frac{\left(P_{t} / P_{t+1}\right)}{\left(C_{t}^{*} / C_{t+1}^{*}\right)}=\left[\frac{\partial\left(C_{t}^{*} / C_{t+1}^{*}\right)}{\partial\left(P_{t}^{*} / P_{t+1}^{*}\right)} \frac{d\left(P_{t}^{*} / P_{t+1}^{*}\right)}{d\left(P_{t} / P_{t+1}\right)}\right] \frac{\left(P_{t} / P_{t+1}\right)}{\left(C_{t} / C_{t+1}\right)}=-\gamma(1-a)
$$

Elasticidade do gasto em relação à $P_{t}-$ efeito intratemporal:

$$
\frac{d C_{t}}{d P_{t}} \frac{P_{t}}{C_{t}}=\left[\frac{\partial C_{t}}{\partial P_{t}^{*}} \frac{d P_{t}^{*}}{d P_{t}}\right] \frac{P_{t}}{C_{t}}=(1-a)
$$




\section{REFERÊNCIAS BIBLIOGRÁFICAS}

Araújo, Carlos Hamilton V.; da Silveira Filho, Getúlio B. Mudanças de Regime no Câmbio Brasileiro. Brasília, jun. 2002. 1-46 p. (Trabalho para discussão, 41).

Barro, Robert; Sala-I-Martin, Xavier. World real interest rates. In: Blanchard, Olivier; Fischer, Stanley (Ed.). NBER Macroeconomics annual 1990. Cambridge: MIT Press, 1990. p. 15-61.

Bergin, Paul R.; Sheffrin, Steven M. Interest rates, exchange rates and present value models of the current account. Economic Journal, v. 110, n. 463, p. 535-58, Apr. 2000.

Campbell, John Y. Does saving anticipate declining labor income? an alternative test of the permanent income hypothesis. Econometrica, v. 55, p. 1249-1273, nov. 1987.

Campbell, John Y.; Lo, Andrew W.; Mackinlay, A. Craig. The Econometrics of Financial Markets. Princeton, New Jersey: Princeton University Press, 1997.

Campbell, John Y.; Mankiw, N. Gregory. Consumption, income, and interest rates: Reinterpreting the time series evidence. In: Blanchard, Olivier; Fischer, Stanley (Ed.). NBER Macroeconomics Annual 1989. Cambridge: MIT Press, 1989. p. 185-216.

Campbell, John Y; Shiller, Robert J. Cointegration and tests of present value models. Journal of Political Economy, v. 95, p. 1062-88, 1987.

Dornbusch, Rudiger. Real interest rates, home goods, and optimal external borrowing. Journal of Political Economy, v. 91, n. 1, p. 141-153, Nov. 1983.

Ghosh, Atish R. International capital mobility amongst the major industrialized countries: Too little or too much? The Economic Journal, v. 105, n. 428, p. 107-128, Jan. 1995.

Grossman, Sanford J.; Shiller, Robert J. The determinants of the variability of stock market prices. American Economic Review, v. 71, n. 2, p. 222-227, 1981.

Hall, Robert E. Intertemporal substitution in consumption. Journal of Political Economy, v. 96, n. 2, p. 339-357, Apr. 1988.

Hansen, Lars Peter; Singleton, Kenneth J. Stochastic consumption, risk aversion, and the temporal behavior of asset returns. Journal of Political Economy, v. 91, n. 2, p. 249-65, Apr. 1983.

Huang, Chao-Hsi; LIN, Kenneth S. Deficits, government expenditures, and tax smoothing in the united states: 1929-1988. Journal of Monetary Economics, v. 31, n. 3, p. 317-339, Jun. 1993.

Iscan, Talan B. Present value tests of the current account with durables consumption. Journal of International Money and Finance, v. 21, n. 3, p. 385-412, Jun. 2002.

Issler, João Victor; Piqueira, Natalia Scotto. Estimating relative risk aversion, the discount rate, and the intertemporal elasticity of substitution in consumption for brazil using three types of utility function. Brazilian Review of Econometrics, v. 20, n. 2, p. 200-238, 2000.

Kravis, Irving B.; Heston, Alan; Summers, Robert. World Product and Income: International Comparisons of Real Gross Product. Baltimore, MD: The Johns Hopkins University Press, 1982.

Mehra, Rajnish; Prescott, Edward C. The equity premium: A puzzle. Journal of Monetary Economics, v. 15, n. 2, p. 145-161, Mar. 1985.

Reis, Eustáquio et al. Renda permanente e poupança precaucional: evidências empíricas para o Brasil no passado recente. Pesquisa e Planejamento Econômico, v. 28, n. 2, p. 233-271, ago. 1998.

Rogoff, Kenneth. Traded goods consumption smoothing and the random walk behavior of the real exchange rate. Bank of Japan Monetary and Economic Studies, v. 10, n. 2, p. 1-29, Nov. 1992.

Senna, Fernanda Assed de A.; Issler, João Victor. Mobilidade de capitais e movimentos da conta corrente do Brasil: 1947-1997. Estudos Econômicos, v. 30, n. 4, p. 493-523, 2000. 
Sheffrin, Steven M.; Woo, Wing Thye. Present value tests of an intertemporal model of the current account. Journal of International Economics, v. 29, n. 3-4, p. 237-253, Nov. 1990.

Silva, Nelson da; Andrade, Joaquim Pinto de. Dinâmica das transações correntes do Brasil: Avalição do modelo básico da abordagem intertemporal. Pesquisa e Planejamento Econômico, v. 36, n. 3, p. 525-550, dez. 2006.

Stockman, Alan C.; Tesar, Linda L. Tastes and technology in a two-country model of the business cycle: Explaining international comovements. American Economic Review, v. 85, n. 1, p. 168-185, Mar. 1995. 